\title{
DECELERATION OF ALPHA PARTICLES IN THE SOLAR WIND BY INSTABILITIES AND THE ROTATIONAL FORCE: IMPLICATIONS FOR HEATING, AZIMUTHAL FLOW, AND THE PARKER SPIRAL MAGNETIC FIELD
}

\author{
Daniel Verscharen ${ }^{1}$, Benjamin D. G. Chandran ${ }^{1,2}$, Sofiane Bourouaine ${ }^{1}$, and Joseph V. Hollweg ${ }^{1}$ \\ ${ }^{1}$ Space Science Center, University of New Hampshire, Durham, NH 03824, USA; daniel.verscharen@unh.edu, \\ benjamin.chandran@unh.edu, s.bourouaine@unh.edu, joe.hollweg@unh.edu \\ ${ }^{2}$ Department of Physics, University of New Hampshire, Durham, NH 03824, USA \\ Received 2014 November 17; accepted 2015 March 1; published 2015 June 15
}

\begin{abstract}
Protons and alpha particles in the fast solar wind are only weakly collisional and exhibit a number of nonequilibrium features, including relative drifts between particle species. Two non-collisional mechanisms have been proposed for limiting differential flow between alpha particles and protons: plasma instabilities and the rotational force. Both mechanisms decelerate the alpha particles. In this paper, we derive an analytic expression for the rate $Q_{\text {flow }}$ at which energy is released by alpha-particle deceleration, accounting for azimuthal flow and conservation of total momentum. We show that instabilities control the deceleration of alpha particles at $r<r_{\text {crit }}$, and the rotational force controls the deceleration of alpha particles at $r>r_{\text {crit }}$, where $r_{\text {crit }} \simeq 2.5 \mathrm{AU}$ in the fast solar wind in the ecliptic plane. We find that $Q_{\text {flow }}$ is positive at $r<r_{\text {crit }}$ and $Q_{\text {flow }}=0$ at $r \geqslant r_{\text {crit }}$, consistent with the previous finding that the rotational force does not lead to a release of energy. We compare the value of $Q_{\text {flow }}$ at $r<r_{\text {crit }}$ with empirical heating rates for protons and alpha particles, denoted $Q_{\mathrm{p}}$ and $Q_{\alpha}$, deduced from in situ measurements of fast-wind streams from the Helios and Ulysses spacecraft. We find that $Q_{\text {flow }}$ exceeds $Q_{\alpha}$ at $r<1 \mathrm{AU}$, and that $Q_{\text {flow }} / Q_{\mathrm{p}}$ decreases with increasing distance from the Sun from a value of about one at $r=0.29-0.42$ AU to about $1 / 4$ at 1 AU. We conclude that the continuous energy input from alpha-particle deceleration at $r<r_{\text {crit }}$ makes an important contribution to the heating of the fast solar wind. We also discuss the implications of the alpha-particle drift for the azimuthal flow velocities of the ions and for the Parker spiral magnetic field.
\end{abstract}

Key words: instabilities - plasmas - solar wind - Sun: corona - turbulence - waves

\section{INTRODUCTION}

The solar wind is a magnetized plasma consisting of protons, electrons, and other ion species. Of the other ion species, alpha particles play the most important role in the overall dynamics and thermodynamics of the solar wind, because they comprise $\sim 15 \%$ of the total solar-wind mass density (Bame et al. 1977; Li et al. 2006; Marsch \& Richter 1984; Pizzo et al. 1983). Observations of protons and alpha particles in the solar wind also show that the temperature profiles of both species decrease more slowly with distance from the Sun than expected in an adiabatically or double-adiabatically (see Chew et al. 1956) expanding gas (Cranmer et al. 2009; Gazis \& Lazarus 1982; Hellinger et al. 2011; Hellinger \& Trávníček 2013; Lamarche et al. 2014; Marsch et al. 1982b, 1982c, 1983, Maruca et al. 2011; Miyake \& Mukai 1987; Schwartz \& Marsch 1983; Thieme et al. 1989). This finding implies that a continuous heating mechanism acts on the solar-wind ions during their transit through the heliosphere. However, there is still no consensus on the mechanisms responsible for this heating.

In the fast solar wind, expansion and heating lead to nonequilibrium features in the distribution functions of the particle species (Goldstein et al. 2000; Kasper et al. 2013; Marsch et al. 1982b, 1982c; Maruca et al. 2012; Reisenfeld et al. 2001) because the collision timescale for ions is typically much larger than the travel time from the Sun (Kasper et al. 2008). These non-equilibrium features include relative drifts between the plasma species along the direction of the magnetic field $\boldsymbol{B}$ and temperature anisotropies with respect to $\boldsymbol{B}$. Because collisions are weak in the fast solar wind, kinetic micro-instabilities are an important process for limiting these deviations from equilibrium (e.g., Gary 1993; Gary et al. 2000b, 2003, Hollweg et al. 2014; Lu et al. 2006). In-situ measurements have shown that the solar wind is confined to regions of parameter space that are bounded by the thresholds of different instabilities (Bale et al. 2009; Bourouaine et al. 2013; Hellinger et al. 2006, 2011; Kasper et al. 2002; Marsch et al. 2004; Maruca et al. 2012; Matteini et al. 2007). Once an instability threshold is crossed, the corresponding instability reduces the deviation from thermodynamic equilibrium by generating plasma waves that interact with particles to reshape their distribution function.

Observations in the fast solar wind show that the absolute value of the typical relative velocity between alpha particles and protons, denoted $\Delta U_{\alpha \mathrm{p}}$, is of order the local Alfvén speed based on the proton mass density $\rho_{\mathrm{p}}$ (Marsch et al. 1982b; Reisenfeld et al. 2001),

$$
v_{\mathrm{A}} \equiv \frac{B}{\sqrt{4 \pi \rho_{\mathrm{p}}}} .
$$

The alpha-to-proton drift excites the fast-magnetosonic/whistler (FM/W) instability (Gary et al. 2000a; Li \& Habbal 2000; Revathy 1978) and the Alfvén/ion-cyclotron (A/IC) instability (Verscharen et al. 2013b) when the drift velocity $\gtrsim v_{\mathrm{A}}$. The Alfvén speed decreases with distance from the Sun, and thus instabilities continuously decelerate the alpha particles (provided that $r$ is not too large, as we will show in this paper). Previous studies have discussed the energy that is available in the relative drift and have suggested that the release of this energy by instabilities in the form of waves makes a significant 
contribution to solar-wind heating (Borovsky \& Gary 2014; Feldman 1979; Šafránková et al. 2013; Schwartz et al. 1981).

The rotational force is another collisionless mechanism that reduces the relative drift speed between protons and alpha particles (Hollweg \& Isenberg 1983; Li \& Li 2006; Li et al. 2007; McKenzie et al. 1979). Roughly speaking, alpha particles and minor ions can be viewed as beads sliding on a wire, where the wire is the spiral interplanetary magnetic field, which is anchored to and rotates with the Sun. Ions with radial velocities $<U_{\mathrm{p} r}$ are accelerated outward by the forces exerted by the rotating "wire," where $U_{\mathrm{p} r}$ is the average proton radial velocity. In contrast, ions with radial velocities exceeding $U_{\mathrm{p} r}$ are decelerated by the rotating wire (Hollweg \& Isenberg 1981; McKenzie et al. 1979). This process is net-energy-conserving and does not release energy that would become available for particle heating.

The central goal of this study is to calculate analytically the rate $Q_{\text {flow }}$ at which energy is released by alpha-particle deceleration, accounting for azimuthal flow and the spiral geometry of the interplanetary magnetic field. We also develop a solar-wind model that allows us to evaluate $Q_{\text {flow }}$ at $0.29 \mathrm{AU}<r<4.2 \mathrm{AU}$. In constructing this model, we draw upon our recent work, in which we derived analytic expressions for the thresholds of the A/IC and FM/W instabilities (Verscharen et al. 2013a). We then compare our solution for $Q_{\text {flow }}(r)$ with the heating rates that are required to explain the observed temperature profiles of protons and alpha particles. For this comparison, we do not discuss the nature of the mechanism that converts $Q_{\text {flow }}$ into particle heating, but rather restrict ourselves to a discussion of the energy available for particle heating. As a by-product of our calculation, we revisit the calculation of the Parker spiral magnetic field and show how the inclusion of differentially flowing alpha particles and the neglect of torque beyond the effective co-rotation point at radius $r_{\mathrm{eff}}$, which is of order the Alfvén critical radius $r_{\mathrm{A}}$, lead to minor modifications to Parker's (1958) original treatment.

We also describe how instabilities and the rotational force work in concert to decelerate the alpha particles. We show that, when the azimuthal velocity is properly included, $Q_{\text {flow }}>0$ at $r<1$ AU and $Q_{\text {flow }} \rightarrow 0$ as $r$ increases to a critical radius $r_{\text {crit }}$. In the fast solar wind, $r_{\text {crit }} \simeq 2.5 \mathrm{AU}$ in the plane of the Sun's equator, and $r_{\text {crit }}$ increases with increasing heliographic latitude $\lambda$. At $r<r_{\text {crit }}$, instabilities are the most efficient deceleration mechanism, and $\Delta U_{\alpha \mathrm{p}}$ is comparable to the threshold drift velocity needed to excite the FM/W instability. At $r>r_{\text {crit }}$, the rotational force is the most efficient deceleration mechanism, the rotational force causes $\Delta U_{\alpha p}$ to become too small to excite instabilities, and $Q_{\text {flow }}=0$. We also show that the condition $Q_{\text {flow }}=0$ leads to the same equation for alpha-particle (and minor-ion) deceleration found in previous studies of the rotational force (Hollweg \& Isenberg 1981; McKenzie et al. 1979), provided that $r$ is sufficiently large that other forces such as gravity can be neglected.

We do not address the details of the solar-wind acceleration mechanisms that lead to a preferential acceleration and heating of the alpha particles close to the Sun. Instead, we assume that one or more mechanisms "charge" an energy source similar to a battery in the very inner heliosphere by preferentially accelerating the alpha particles, and that this source is then continuously "discharged" by the deceleration of the alpha particles by micro-instabilities. Candidate mechanisms for generating alpha-particle beams in the solar wind include cyclotron-resonant wave-particle interactions (Dusenbery \& Hollweg 1981; Hollweg \& Isenberg 2002; Isenberg \& Vasquez 2007, 2009; Marsch et al. 1982a; McKenzie \& Marsch 1982; Ofman et al. 2002), the dissipation of low-frequency waves in an inhomogeneous plasma (Isenberg \& Hollweg 1982; McKenzie et al. 1979), and stochastic heating by lowfrequency turbulence (Chandran 2010; Chandran et al. 2010, 2013; Chaston et al. 2004; Chen et al. 2001; Johnson \& Cheng 2001; McChesney et al. 1987).

The remainder of this paper is organized as follows. In Section 2, we derive an analytic expression for $Q_{\text {flow }}$, taking into account the azimuthal velocities of the ions. In Section 3, we develop a solar-wind model that accounts for azimuthal flow, the spiral interplanetary magnetic field, and the deceleration of alpha particles by plasma instabilities and the rotational force. In Section 4, we present numerical solutions to our model equations at zero heliographic latitude for heliocentric distances in the range $0.29 \mathrm{AU}<r<1 \mathrm{AU}$, and we compare our results with measurements from the Helios spacecraft. In Section 5, we present numerical solutions at a range of heliographic latitudes for heliocentric distances in the range 1.5 AU $<r<4.2 \mathrm{AU}$, and we compare our results with measurements from the Ulysses spacecraft. In Section 6, we justify our approximation of neglecting the net force on the solar wind at $r>0.29 \mathrm{AU}$. We summarize our conclusions in Section 7, and in the Appendix we discuss the sensitivity of the FM/W and A/IC instability thresholds to the alpha-particle temperature anisotropy.

\section{THE HEATING POWER THAT RESULTS FROM ALPHA-PARTICLE DECELERATION}

We work in a non-rotating reference frame and use heliocentric spherical coordinates $(r, \theta, \phi)$, where the $\theta=0$ direction is aligned with the Sun's angular-momentum vector. We assume cylindrical symmetry and steady-state conditions,

$$
\frac{\partial}{\partial \phi}=\frac{\partial}{\partial t}=0
$$

and we set

$$
U_{j \theta}=0
$$

We restrict our analysis to heliocentric distances $>0.29 \mathrm{AU}$, so that the net force on the solar wind can be neglected to a reasonable approximation. We discuss this "coasting approximation" further in Section 6.

Upon summing the radial and azimuthal components of the momentum equation for all particle species, invoking the "coasting approximation," and making use of Equations (2) and (3), we obtain

$$
\sum_{j}\left[\rho_{j} U_{j r} \frac{\partial U_{j r}}{\partial r}-\frac{\rho_{j} U_{j \phi}^{2}}{r}\right]=0
$$

and

$$
\sum_{j}\left[\rho_{j} U_{j r} \frac{\partial U_{j \phi}}{\partial r}+\frac{\rho_{j} U_{j r} U_{j \phi}}{r}\right]=0,
$$

where $\boldsymbol{U}_{j}\left(\rho_{j}\right)$ is the velocity (mass density) of species $j$. For protons $j=\mathrm{p}$, and for alpha particles $j=\alpha$. The contribution 
of electrons to the momentum density is negligible due to their small mass. Given Equations (2) and (3), mass conservation requires that

$$
\frac{1}{r^{2}} \frac{\partial}{\partial r}\left(r^{2} \rho_{j} U_{j r}\right)=0
$$

for each particle species.

We neglect finite-Larmor-radius corrections, and thus the relative drift of alpha particles with respect to the protons is aligned with the magnetic field $\boldsymbol{B}$. For concreteness, we take

$$
B_{r}>0 \quad \text { and } \quad B_{\phi}<0 \text {, }
$$

where the second inequality follows from the first because field lines "bend back" in the $-\widehat{\phi}$ direction as the Sun rotates in the $+\widehat{\phi}$ direction. Because of Equation (7), we adopt the convention that the angle $\psi_{B}$ between $\boldsymbol{B}$ and $\widehat{\boldsymbol{r}}$ is negative (or at least non-positive):

$$
\psi_{B} \leqslant 0 .
$$

Thus,

$$
U_{\alpha r}=U_{\mathrm{p} r}+\Delta U_{\alpha \mathrm{p}} \cos \psi_{B}
$$

and

$$
U_{\alpha \phi}=U_{\mathrm{p} \phi}+\Delta U_{\alpha \mathrm{p}} \sin \psi_{B} .
$$

The rate $Q_{\text {flow }}$ at which bulk-flow kinetic energy is converted into other forms of energy is given by the negative of the divergence of the kinetic-energy flux. Making use of Equations (2) and (3), we can write

$$
Q_{\text {flow }}=-\sum_{j}\left[\frac{1}{r^{2}} \frac{\partial}{\partial r}\left(r^{2} \frac{\rho_{j} U_{j}^{2}}{2} U_{j r}\right)\right],
$$

where $U_{j}^{2}=U_{j r}^{2}+U_{j \phi}^{2}$. The energy that is taken out of the bulk flow is transformed into waves and thermal energy. Since the waves cascade and dissipate, we expect that $Q_{\text {flow }}$ is in effect the heating rate that results from alpha-particle deceleration.

With the use of Equations (4) and (10), we express the gradient of $U_{\mathrm{p} r}$ in terms of the gradient of the relative drift $\Delta U_{\alpha \mathrm{p}}$ :

$$
\begin{gathered}
\frac{\partial U_{\mathrm{p} r}}{\partial r}=(\mu-1) \frac{\partial}{\partial r}\left(\Delta U_{\alpha \mathrm{p}} \cos \psi_{B}\right) \\
+\frac{\mu\left(\rho_{\mathrm{p}} U_{\mathrm{p} \phi}^{2}+\rho_{\alpha} U_{\alpha \phi}^{2}\right)}{\rho_{\mathrm{p}} U_{\mathrm{p} r} r},
\end{gathered}
$$

where

$$
\mu \equiv \frac{\rho_{\mathrm{p}} U_{\mathrm{p} r}}{\rho_{\mathrm{p}} U_{\mathrm{p} r}+\rho_{\alpha} U_{\alpha r}}
$$

is of order unity in the solar wind. Because of Equation (6),

$$
\frac{\partial \mu}{\partial r}=0
$$

From Equation (9), we see that $(\partial / \partial r) U_{\alpha r}$ is given by the righthand side of Equation (12) replacing $(\mu-1)$ in the first term on the right-hand side with just $\mu$. Likewise, with the use of Equations (5) and (10), we find that

$$
\begin{aligned}
\frac{\partial U_{\mathrm{p} \phi}}{\partial r}= & (\mu-1) \frac{\partial}{\partial r}\left(\Delta U_{\alpha \mathrm{p}} \sin \psi_{B}\right) \\
& -\frac{\mu\left(\rho_{\mathrm{p}} U_{\mathrm{p} r} U_{\mathrm{p} \phi}+\rho_{\alpha} U_{\alpha r} U_{\alpha \phi}\right)}{\rho_{\mathrm{p}} U_{\mathrm{p} r} r} .
\end{aligned}
$$

From Equation (10), we see that $(\partial / \partial r) U_{\alpha \phi}$ is given by the right-hand side of Equation $(15)$ replacing $(\mu-1)$ in the first term on the right-hand side with just $\mu$.

Now that we have expressed the radial derivatives of $U_{\mathrm{p} r}$, $U_{\mathrm{p} \phi}, U_{\alpha r}$, and $U_{\alpha \phi}$ in terms of $(\partial / \partial r) \Delta U_{\alpha \mathrm{p}}$, we can re-express $Q_{\text {flow }}$ in the form

$$
\begin{aligned}
Q_{\text {flow }}= & -\mu \rho_{\alpha} U_{\alpha r} \frac{\partial}{\partial r} \frac{\left(\Delta U_{\alpha \mathrm{p}}\right)^{2}}{2} \\
& -\frac{\mu \rho_{\alpha}\left(U_{\mathrm{p} r} U_{\alpha \phi}-U_{\alpha r} U_{\mathrm{p} \phi}\right)^{2}}{r U_{\mathrm{p} r}} .
\end{aligned}
$$

Because we neglect resistivity and finite-Larmor-radius corrections, the magnetic field is frozen to each particle species. In the reference frame that co-rotates with the Sun, the magnetic field lines are thus parallel to both $\boldsymbol{U}_{\mathrm{p}}$ and $\boldsymbol{U}_{\alpha}$ (Mestel 1968). This leads to

$$
\tan \psi_{B}=\frac{U_{\mathrm{p} \phi}}{U_{\mathrm{p} r}}-\frac{\Omega_{\odot} r \sin \theta}{U_{\mathrm{p} r}}=\frac{U_{\alpha \phi}}{U_{\alpha r}}-\frac{\Omega_{\odot} r \sin \theta}{U_{\alpha r}} .
$$

(We note that the second equality in Equation (17) follows from the first equality with the use of Equations (9) and (10), which is just the condition that $\boldsymbol{U}_{\alpha}-\boldsymbol{U}_{\mathrm{p}}$ is parallel to $\boldsymbol{B}$.) With these expressions for $U_{\mathrm{p} \phi}$ and $U_{\alpha \phi}$, we can rewrite Equation (16) as

$$
\begin{aligned}
Q_{\text {flow }}= & -\mu \rho_{\alpha}\left[U_{\alpha r} \frac{\partial}{\partial r} \frac{\left(\Delta U_{\alpha \mathrm{p}}\right)^{2}}{2}\right. \\
& \left.+\frac{r\left(\Omega_{\odot} \sin \theta\right)^{2}\left(\Delta U_{\alpha \mathrm{p}} \cos \psi_{B}\right)^{2}}{U_{\mathrm{p} r}}\right] .
\end{aligned}
$$

\section{SOLAR-WIND MODEL WITH AZIMUTHAL VELOCITIES AND DIFFERENTIAL FLOW}

In this section, we expand upon the assumptions made in Section 2 to develop a model of the solar wind that will enable us to evaluate $Q_{\text {flow }}$ as a function of $r$. This model can be viewed as consisting of four equations for four unknowns: $U_{\mathrm{p} r}$, $U_{\mathrm{p} \phi}, \psi_{B}$, and $\Delta U_{\alpha \mathrm{p}}$. The alpha-particle velocity components $U_{\alpha r}$ and $U_{\alpha \phi}$ can be trivially obtained from these quantities using Equations (9) and (10).

The first of the four equations in our model is Equation (4), the radial component of the total-momentum equation. Because we work in the "coasting approximation," Equation (4) neglects the plasma pressure, the pressure associated with waves and turbulence, and gravity, which is reasonable given 
that we focus on heliocentric distances $>0.29 \mathrm{AU}$ (see Section 6).

The second of the four equations in our solar-wind model is Equation (5), the $\phi$ component of the total-momentum equation, which we rewrite as follows. First, we integrate Equation (5) to obtain an equation that expresses angularmomentum conservation:

$$
\mathcal{F} \equiv r^{3} \rho_{\mathrm{p}} U_{\mathrm{p} r} U_{\mathrm{p} \phi}+r^{3} \rho_{\alpha} U_{\alpha r} U_{\alpha \phi}=\text { constant }
$$

(i.e., $\partial \mathcal{F} / \partial r=0)$, where $2 \pi \mathcal{F} \sin \theta d \theta$ is the rate at which angular momentum flows out through radius $r$ between spherical polar angles $\theta$ and $\theta+d \theta$. We note that Equation (6) implies that

$$
\mathcal{G}_{j} \equiv r^{2} \rho_{j} U_{j r}=\text { constant }
$$

(i.e., $\left.\partial \mathcal{G}_{j} / \partial r=0\right)$. We then rewrite Equation (19) using Equations (10) and (20) to eliminate $U_{\alpha r}$ and $U_{\alpha \phi}$, obtaining

$$
U_{\mathrm{p} \phi}=\frac{\mathcal{F}}{\left(\mathcal{G}_{\mathrm{p}}+\mathcal{G}_{\alpha}\right) r}+(\mu-1) \Delta U_{\alpha \mathrm{p}} \sin \psi_{B}
$$

Close to the Sun, the Lorentz force exerts a non-negligible torque on the solar wind. This torque gradually decreases with distance from the Sun, and the solar wind behaves like a nettorque-free plasma outflow at large $r$. The azimuthal velocity profiles at large $r$ can be approximated as the result of a plasma flow that is co-rotating out to a certain distance and then torque-free beyond this distance. We define this distance from the Sun as the effective co-rotation radius $r_{\text {eff }}$, which is of order the Alfvén critical radius $r_{\mathrm{A}}$ (cf Hollweg \& Lee 1989). We assume that at $r=r_{\mathrm{eff}}, \psi_{B}=0$ and the protons and alpha particles co-rotate with the Sun: $U_{\mathrm{p} \phi}\left(r_{\mathrm{eff}}\right)=U_{\alpha \phi}\left(r_{\mathrm{eff}}\right)=$ $\Omega_{\odot} r_{\text {eff }} \sin \theta$. This allows us to rewrite Equation (21) as

$$
U_{\mathrm{p} \phi}=\frac{\Omega_{\odot} r_{\mathrm{eff}}^{2} \sin \theta}{r}+(\mu-1) \Delta U_{\alpha \mathrm{p}} \sin \psi_{B} .
$$

In the numerical calculations below, we set $r_{\text {eff }}=10 R_{\odot}$.

The third of the four equations in our model is Equation (17), which expresses the condition that the proton and alpha-particle velocities are parallel to $\boldsymbol{B}$ in the reference frame that co-rotates with the Sun. With the help of Equation (22), we rewrite Equation (17) as

$$
\tan \psi_{B}+(1-\mu) \frac{\Delta U_{\alpha \mathrm{p}}}{U_{\mathrm{p} r}} \sin \psi_{B}=\frac{\Omega_{\odot} \sin \theta}{r U_{\mathrm{p} r}}\left(r_{\mathrm{eff}}^{2}-r^{2}\right) .
$$

As we will discuss further in Section 4.2, Equation (23) is similar to Parker's (1958) equation for the spiral interplanetary magnetic field (see Equation (53)). However, a new feature of Equation (23) is the appearance of the second term on the lefthand side, which describes the effects of differential flow on the angle $\psi_{B}$.

The fourth and final equation in our solar-wind model describes the radial evolution of $\Delta U_{\alpha \mathrm{p}}$. We explain how we obtain this fourth equation in Section 3.1.

\subsection{Determination of $\Delta U_{\alpha \mathrm{p}}$}

We consider two non-collisional mechanisms that decelerate alpha particles in the solar wind: plasma instabilities and the rotational force. ${ }^{3}$ We neglect the collisional deceleration of alpha particles with respect to the protons because the collisional mean free path is large $(\gtrsim r)$ in the fast solar wind at $r \gtrsim 0.29$ AU. We discuss instabilities in Section 3.1.1, the rotational force in Section 3.1.2, and the combined effects of both mechanisms in Section 3.1.3.

\subsubsection{Instability Thresholds}

In this paper, we focus on heliocentric distances $r \gtrsim 0.29 \mathrm{AU}$, at which $\beta$ (the ratio of plasma pressure to magnetic pressure) is typically $\gtrsim 0.2$ (see Figure 4 ). When $\beta \gtrsim 0.2$, the plasma instabilities that are most easily excited by the differential flow between alpha particles and protons are the parallel-propagating FM/W mode and the parallel-propagating A/IC mode (Gary et al. 2000b, 2000a; Li \& Habbal 2000; Scarf $\&$ Fredricks 1968; Verscharen et al. 2013b, 2013a). (In contrast, at smaller values of $\beta$, oblique $\mathrm{A} / \mathrm{IC}$ modes are more easily excited than these parallel modes (Gary et al. 2000b; Verscharen \& Chandran 2013).) The characteristic value of $\Delta U_{\alpha \mathrm{p}}$ at which the FM/W and $\mathrm{A} / \mathrm{IC}$ modes become unstable is $\sim v_{\mathrm{A}}$. However, as shown by Revathy (1978), Araneda et al. (2002), Gary et al. (2003), and Verscharen et al. (2013a), a temperature anisotropy of the form $T_{\perp \alpha}>T_{\| \alpha}$ reduces the minimum value of $\Delta U_{\alpha \mathrm{p}}$ needed to excite the $\mathrm{A} / \mathrm{IC}$ instability, while a temperature anisotropy of the form $T_{\perp \alpha}<T_{\| \alpha}$ reduces the minimum value of $\Delta U_{\alpha \mathrm{p}}$ needed to excite the FM/W instability, where $T_{\perp \alpha}\left(T_{\| \alpha}\right)$ is the alpha-particle temperature perpendicular (parallel) to $\boldsymbol{B}$.

When one of these instability thresholds is crossed, resonant wave-particle interactions cause the corresponding plasma wave $(\mathrm{A} / \mathrm{IC}$ or $\mathrm{FM} / \mathrm{W})$ to grow and the drift velocity and/or temperature anisotropy to decrease. The characteristic time scales on which instabilities grow and reduce $\Delta U_{\alpha p}$ in the solar wind are much smaller than the time scales associated with changes in the background parameters. Therefore, if some mechanism (e.g., the radial decrease in $v_{\mathrm{A}}$ ) drives the plasma toward the unstable region of parameter space, then instabilities rapidly push the plasma back toward the instability threshold, holding the plasma in a marginally stable state until some other mechanism (such as the rotational force) reduces $\Delta U_{\alpha \mathrm{p}}$ below the instability threshold.

Verscharen et al. (2013a) derived analytical instability thresholds for the parallel $\mathrm{A} / \mathrm{IC}$ and $\mathrm{FM} / \mathrm{W}$ modes in the presence of alpha-particle temperature anisotropy under the assumption that the alpha particles have a bi-Maxwellian distribution. They found that the minimum value of $\Delta U_{\alpha \mathrm{p}}$ needed to excite the $\mathrm{A} / \mathrm{IC}$ mode is given by

$$
U_{\mathrm{t} 1}=v_{\mathrm{A}}-\sigma_{1}\left(\frac{T_{\perp \alpha}}{T_{\| \alpha}}-1\right) w_{\| \alpha}-\frac{v_{\mathrm{A}}^{2} T_{\| \alpha}}{4 \sigma_{1} w_{\| \alpha} T_{\perp \alpha}}
$$

and the minimum value of $\Delta U_{\alpha \mathrm{p}}$ needed to excite the FM/W instability is given by

$$
U_{\mathrm{t} 2}=v_{\mathrm{A}}-\sigma_{2}\left(1-\frac{T_{\perp \alpha}}{T_{\| \alpha}}\right) w_{\| \alpha}+\frac{v_{\mathrm{A}}^{2} T_{\| \alpha}}{4 \sigma_{2} w_{\| \alpha} T_{\perp \alpha}},
$$

\footnotetext{
3 Wave-pressure forces can also reduce $\Delta U_{\alpha \mathrm{p}}$ (Barnes 1981; Goodrich 1978 Hollweg 1974; Isenberg \& Hollweg 1983), but we focus on heliocentric distances that are sufficiently large that these forces can be neglected.
} 


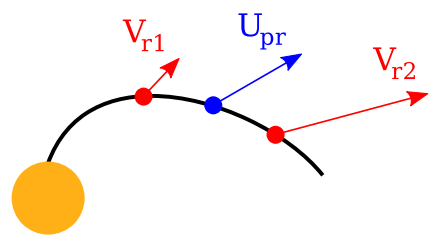

Figure 1. Illustration of the rotational force for protons (blue dot) moving outwards with velocity $U_{\mathrm{p} r}$ and two test particles (red dots) with different radial velocities $V_{r}\left(V_{r 1}<U_{\mathrm{p} r}\right.$ and $\left.V_{r 2}>U_{\mathrm{p} r}\right)$. These test particles behave like beads sliding on a frictionless wire, where the wire is the spiral magnetic field.

where

$$
w_{\| \alpha} \equiv \sqrt{\frac{2 k_{\mathrm{B}} T_{\| \alpha}}{m_{\alpha}}}
$$

is the parallel thermal speed of the alpha particles,

$$
\sigma_{i} \equiv \sqrt{-\ln \frac{M_{i} n_{\mathrm{p}}}{n_{\alpha}}},
$$

the subscript $i=1$ corresponds to the A/IC mode, the subscript $i=2$ corresponds to the FM/W mode, $M_{1}=1.6 \times 10^{-4}$, $M_{2}=6.1 \times 10^{-4}$, and $n_{\alpha}$ and $n_{\mathrm{p}}$ are, respectively, the number densities of the alpha particles and protons. These choices for the parameters $M_{1}$ and $M_{2}$ lead to a maximum growth rate of $\gamma_{\mathrm{m}}=10^{-4} \Omega_{\mathrm{p}}$ for the corresponding instability. For further details, we refer the reader to the original publication (Verscharen et al. 2013a).

As discussed by Verscharen et al. (2013b), the A/IC instability is driven by resonant alpha particles whose outward velocities are smaller than $U_{\mathrm{p} r}$ - that is, alpha particles that flow toward the Sun in the proton frame. It is thus not clear how the A/IC instability could decelerate the bulk of the alpha-particle population in the solar wind. On the other hand, the FM/W instability resonates with individual alpha particles whose outward velocities exceed a certain threshold of order $U_{\mathrm{p}}+v_{\mathrm{A}}$ (see discussion by Verscharen et al. 2013a). We thus expect that it is the FM/W instability and not the A/IC instability that leads to the ongoing deceleration of alpha particles in the solar wind, even if the A/IC instability has a lower threshold under the assumption of bi-Maxwellian particle distributions. Thus, when the alpha-proton drift is limited by instabilities, we set

$$
\Delta U_{\alpha \mathrm{p}}=U_{\mathrm{t} 2} .
$$

For a discussion of other beam-driven instabilities, we refer the reader to Gary et al. (2000b), Verscharen \& Chandran (2013), and Hollweg et al. (2014).

\subsubsection{The Rotational Force}

A second mechanism that decelerates alpha particles in the solar wind is the rotational force (Hollweg \& Isenberg 1981, 1983; McKenzie et al. 1979; McKenzie \& Axford 1983). The basic idea behind the rotational force can be understood with the aid of Figure 1: at least for the special (hypothetical) case in which $U_{\mathrm{p} r}$ is constant, all ion species besides protons have negligible densities, and $U_{\mathrm{p} \phi}=0$. (These restrictions are not made in the analysis below.) Because the protons are frozen to the interplanetary magnetic field, the Sun's rotation coupled with the protons' radial motion causes the magnetic field to follow a spiral pattern, as first described by Parker (1958). The behavior of any individual charged test particle can then be understood by viewing the particle as a bead sliding along a frictionless wire, where the role of the wire is played by the magnetic field lines, which rotate with the Sun. Any test particle with a radial velocity smaller than $U_{\mathrm{p} r}$ behaves like a bead that is initially at rest: it is flung outward by the forces resulting from the wire's rotation. On the other hand, a test particle with radial velocity exceeding $U_{\mathrm{p} r}$ experiences the opposite effect: it is decelerated as it moves along the rotating field lines.

To explain this effect, we recount the derivation of the rotational force given by Hollweg \& Isenberg (1981), who analyzed the motion of cold ions and worked in a reference frame that co-rotates with the Sun. (The original derivation by McKenzie et al. (1979) was carried out in a non-rotating frame.) In order to maintain completeness of the discussion of the rotational force, we include gravity in this section. Hollweg \& Isenberg (1981) noted that conservation of energy for the protons implies that

$$
v_{\| \mathrm{p}}^{2}=E_{\mathrm{p}}+\frac{2 G M_{\odot}}{r}+\left(\Omega_{\odot} r \sin \theta\right)^{2}
$$

where $v_{\| \mathrm{p}}$ is the proton velocity in the co-rotating frame, $G$ is the gravitational constant, $M_{\odot}$ is the mass of the Sun, and $E_{\mathrm{p}}$ is a constant related to the total proton energy. The notation $v_{\| \mathrm{p}}$ (and $v_{\| \alpha}$ below) is used because, as discussed above, in the corotating frame both the protons and the alpha particles flow parallel to the magnetic field. Conservation of energy for the alpha particles implies that

$$
v_{\| \alpha}^{2}=E_{\alpha}+\frac{2 G M_{\odot}}{r}+\left(\Omega_{\odot} r \sin \theta\right)^{2},
$$

where $E_{\alpha}$ is a constant. We have trivially generalized Hollweg \& Isenberg's (1981) original expressions by allowing $\theta$ to differ from $\pi / 2$. Subtracting Equation (29) from Equation (30) yields

$$
v_{\| \alpha}-v_{\| \mathrm{p}}=\frac{E_{\alpha}-E_{\mathrm{p}}}{v_{\| \alpha}+v_{\| \mathrm{p}}} .
$$

Equations (29) and (30) lead to the asymptotic scaling $v_{\| \mathrm{p}} \propto v_{\| \alpha} \propto r$ at large $r$ (provided $\sin \theta \neq 0$, so that rotation is relevant). At large $r$, Equation (31) thus gives

$$
v_{\| \alpha}-v_{\| \mathrm{p}} \propto \frac{1}{r} \quad \text { as } \quad r \rightarrow \infty .
$$

Thus, the difference in the velocities of the two particle species decreases with distance from the Sun.

We now show that Equations (29) and (30), and hence Equations (31) and (32), are equivalent to the condition

$$
Q_{\text {flow }}=0 \text {, }
$$

provided that gravity can be neglected. (McKenzie et al. 1979 argued that gravity can be neglected when treating the rotational force at $r \gtrsim 0.2 \mathrm{AU}$ in the ecliptic plane, and we neglect gravity throughout our analysis; we discuss this approximation further in Section 6.) Equation (33) can be 
rewritten in the form

$$
\frac{1}{2} \rho_{\mathrm{p}} \boldsymbol{U}_{\mathrm{p}} \cdot \nabla U_{\mathrm{p}}^{2}+\frac{1}{2} \rho_{\alpha} \boldsymbol{U}_{\alpha} \cdot \nabla U_{\alpha}^{2}=0 .
$$

Equations (4) and (5), expressing total-momentum conservation, can be written as a single vector equation,

$$
\rho_{\mathrm{p}} \boldsymbol{U}_{\mathrm{p}} \cdot \nabla \boldsymbol{U}_{\mathrm{p}}+\rho_{\alpha} \boldsymbol{U}_{\alpha} \cdot \nabla \boldsymbol{U}_{\alpha}=0
$$

Upon taking the scalar product of Equation (35) with $\boldsymbol{U}_{\mathrm{p}}$ and subtracting the resulting equation from Equation (34), we obtain

$$
\left(\boldsymbol{U}_{\alpha} \cdot \nabla \boldsymbol{U}_{\alpha}\right) \cdot\left(\boldsymbol{U}_{\alpha}-\boldsymbol{U}_{\mathrm{p}}\right)=0
$$

Likewise, upon taking the scalar product of Equation (35) with $\boldsymbol{U}_{\alpha}$ and subtracting the resulting equation from Equation (34), we obtain

$$
\left(\boldsymbol{U}_{\mathrm{p}} \cdot \nabla \boldsymbol{U}_{\mathrm{p}}\right) \cdot\left(\boldsymbol{U}_{\mathrm{p}}-\boldsymbol{U}_{\alpha}\right)=0
$$

In the reference frame that co-rotates with the Sun, both the protons and the alpha particles flow parallel to the magnetic field. Thus, the proton and alpha-particle velocities in the nonrotating frame are related to $v_{\| \mathrm{p}}$ and $v_{\| \alpha}$ through the equations

$$
\boldsymbol{U}_{\mathrm{p}}=v_{\| \mathrm{p}} \hat{\boldsymbol{b}}+\Omega_{\odot} \hat{z} \times \boldsymbol{r}
$$

and

$$
\boldsymbol{U}_{\alpha}=v_{\| \alpha} \hat{\boldsymbol{b}}+\Omega_{\odot} \hat{\boldsymbol{z}} \times \boldsymbol{r},
$$

where $\hat{\boldsymbol{b}}$ is the magnetic-field unit vector, $\Omega_{\odot} \widehat{z}$ is the angular velocity of the Sun, and $\boldsymbol{r}$ is the position vector of the point at which the velocities are being evaluated in a reference frame centered on the Sun. It follows from Equations (38) and (39) that $\boldsymbol{U}_{\alpha}-\boldsymbol{U}_{\mathrm{p}} \propto \hat{\boldsymbol{b}}$, and thus Equations (36) and (37) can be rewritten as

$$
\left(\boldsymbol{U}_{\alpha} \cdot \nabla \boldsymbol{U}_{\alpha}\right) \cdot \hat{\boldsymbol{b}}=0
$$

and

$$
\left(\boldsymbol{U}_{\mathrm{p}} \cdot \nabla \boldsymbol{U}_{\mathrm{p}}\right) \cdot \hat{\boldsymbol{b}}=0
$$

respectively (where we have assumed that $v_{\| \mathrm{p}} \neq v_{\| \alpha}$, so that there is some differential flow). Physically, Equations (40) and (41) state the essence of the "bead-on-wire" approximation: ions (the "beads") can experience forces perpendicular, but not parallel, to the "wire" (the magnetic field). Because of this, we should be able to use Equations (40) and (41) to recover Hollweg \& Isenberg's (1981) results. In fact, all that is required is to substitute Equation (39) into Equation (40) and to substitute Equation (38) into Equation (41). After a little algebra, ${ }^{4}$ this leads to

$$
\hat{\boldsymbol{b}} \cdot \nabla\left(\frac{v_{\| \alpha}^{2}}{2}-\Omega^{2} r^{2} \sin ^{2} \theta\right)=0
$$

\footnotetext{
4 We use the identities $(\hat{\boldsymbol{b}} \cdot \nabla \hat{\boldsymbol{b}}) \cdot \hat{\boldsymbol{b}}=0,(\hat{\phi} \cdot \nabla \hat{\boldsymbol{b}}) \cdot \hat{\boldsymbol{b}}=0,\left[\hat{\boldsymbol{b}} \cdot \nabla\left(\Omega_{\odot} \hat{z} \times\right.\right.$ $\boldsymbol{r})] \cdot \hat{\boldsymbol{b}}=0$, and $\hat{\phi} \cdot \nabla\left(\Omega_{\odot} \hat{z} \times \boldsymbol{r}\right)=-\Omega_{\odot}[\cos (\theta) \hat{\theta}+\sin (\theta) \boldsymbol{r}]$.
}

and

$$
\hat{\boldsymbol{b}} \cdot \nabla\left(\frac{v_{\| \mathrm{p}}^{2}}{2}-\Omega^{2} r^{2} \sin ^{2} \theta\right)=0
$$

Equations (42) and (43) are equivalent to Equations (29) and (30) for the region on which we focus, in which the gravitational force can be neglected to a good approximation.

Like Hollweg \& Isenberg (1981), we have assumed neither that the protons flow radially nor that the alpha-particle mass density is small. We conclude that alpha particles and protons evolving under the influence of the rotational force are described by the conditions of total-momentum conservation (either Equations (4) and (5) or, equivalently, Equation (35)), the condition of parallel flow velocities (either Equation (17) or, equivalently, Equations (38) and (39)), and the condition $Q_{\text {flow }}=0$. This finding explicitly confirms that alpha-particle deceleration by the rotational force releases no net energy for plasma heating. We note that from Equation (18), we can rewrite the expression $Q_{\text {flow }}=0$ as

$$
\frac{\partial}{\partial r} \Delta U_{\alpha \mathrm{p}}=-\frac{\Omega_{\odot}^{2} r \sin ^{2} \theta \cos ^{2} \psi_{B}}{U_{\alpha r} U_{\mathrm{p} r}} \Delta U_{\alpha \mathrm{p}} .
$$

It is worth noting that McKenzie et al. (1979) and Hollweg \& Isenberg (1981) differed in their views on whether the rotational force involves interaction between the particle species (cf Hollweg \& Isenberg 1983; McKenzie \& Axford 1983). The presence or absence of interaction depends upon which reference frame one works in. As noted by Hollweg \& Isenberg (1981), in a frame of reference that co-rotates with the Sun, the ions behave like non-interacting particles. Each ion species flows along the magnetic field lines subject to a fixed centrifugal potential energy, and the total energy of each species in the co-rotating frame is separately conserved. In contrast, in the non-rotating frame used by McKenzie et al. (1979), the sum of the particle energies is conserved (as shown above from the expression $Q_{\text {flow }}=0$ ), but neither the proton energy nor the alpha-particle energy is individually conserved. Likewise, in this non-rotating frame, neither the proton momentum nor the alpha-particle momentum is conserved, but their sum is. Thus, in the non-rotating frame, the "wire" or magnetic field provides a vehicle through which the two particle species can exchange momentum, angular momentum, and energy.

\subsubsection{Putting It All Together: the Combined Action of Instabilities and the Rotational Force}

In the previous subsections, we described two different mechanisms that decelerate alpha particles. In this section, we describe how these mechanisms decelerate alpha particles over some arbitrary interval of heliocentric distances $\left(r_{1}, r_{1}+\Delta r\right)$.

If the plasma is unstable at $r_{1}$, with $\Delta U_{\alpha \mathrm{p}}>U_{\mathrm{t} 2}$, then the FM/W instability grows and interacts with the alpha particles. The growing FM/W fluctuations rapidly reduce $\Delta U_{\alpha \mathrm{p}}$ toward a state of marginal stability, in which $\Delta U_{\alpha \mathrm{p}}=U_{\mathrm{t} 2}$. Unstable states are transient, and thus we neglect the case $\Delta U_{\alpha \mathrm{p}}>U_{\mathrm{t} 2}$ in our steady-state model.

If the plasma is marginally stable at $r_{1}$, with $\Delta U_{\alpha \mathrm{p}}=U_{\mathrm{t} 2}$, then in the absence of instabilities the rotational force acting on its own would cause $\Delta U_{\alpha p}$ to decrease with a radial derivative 
$(\partial / \partial r) \Delta U_{\alpha p}$ given by the right-hand side of Equation (44). If

$$
\frac{\Omega_{\odot}^{2} r \sin ^{2} \theta \cos ^{2} \psi_{B}}{U_{\alpha r} U_{\mathrm{p} r}} \Delta U_{\alpha \mathrm{p}}<\left|\frac{\partial}{\partial r} U_{\mathrm{t} 2}\right|,
$$

then the rotational force on its own would be unable to decelerate the alpha particles sufficiently rapidly to keep $\Delta U_{\alpha \mathrm{p}}$ at or below the threshold for the FM/W instability throughout the interval $\left(r_{1}, r_{1}+\Delta r\right)$. (Here and in Equation (47) below we have made use of the fact that $(\partial / \partial r) U_{\mathrm{t} 2}<0$ over the radial intervals on which we focus.) Therefore, when Equation (45) is satisfied, plasma instabilities maintain the plasma in a marginally stable state between $r_{1}$ and $r_{1}+\Delta r .{ }^{5}$ We note that when Equation (45) is satisfied at $r_{1}$ and $\Delta U_{\alpha \mathrm{p}}=U_{\mathrm{t} 2}$ between $r_{1}$ and $r_{1}+\Delta r$, it can be seen from Equation (18) that

$$
Q_{\text {flow }}>0
$$

between $r_{1}$ and $r_{1}+\Delta r$.

If the plasma is marginally stable at $r_{1}$ but

$$
\frac{\Omega_{\odot}^{2} r \sin ^{2} \theta \cos ^{2} \psi_{B}}{U_{\alpha r} U_{\mathrm{p} r}} \Delta U_{\alpha \mathrm{p}}>\left|\frac{\partial}{\partial r} U_{\mathrm{t} 2}\right|,
$$

then Equation (44) implies that the rotational force on its own reduces $\Delta U_{\alpha \mathrm{p}}$ sufficiently rapidly that the plasma becomes stable between $r_{1}$ and $r_{1}+\Delta r$, so that plasma instabilities cannot be excited. In this case,

$$
Q_{\text {flow }}=0
$$

between $r_{1}$ and $r_{1}+\Delta r$, and $\Delta U_{\alpha \mathrm{p}}$ evolves according to Equation (44). We note that if we were to mistakenly insist that $\Delta U_{\alpha \mathrm{p}}=U_{\mathrm{t} 2}$ between $r_{1}$ and $r_{1}+\Delta r$ when Equation (47) is satisfied, then we would mistakenly conclude from Equation (18) that $Q_{\text {flow }}$ is negative. In other words, to maintain the state $\Delta U_{\alpha \mathrm{p}}=U_{\mathrm{t} 2}$ when Equation (47) is satisfied, energy would have to be supplied to the plasma in order to overcome the rotational force.

Finally, if the plasma is stable at $r_{1}$, with $\Delta U_{\alpha \mathrm{p}}<U_{\mathrm{t} 2}$, then the FM/W instability is not excited, and the radial evolution of the differential flow between $r_{1}$ and $r_{1}+\Delta r$ is governed by the rotational force. In this case, $(\partial / \partial r) \Delta U_{\alpha p}$ is given by Equation (44), and $Q_{\text {flow }}=0$.

For the numerical solutions that we describe later in this paper, instabilities control the deceleration of the alpha particles at $r<r_{\text {crit }}$, where the critical radius $r_{\text {crit }}$ is $\simeq 2.5 \mathrm{AU}$ in the plane of the ecliptic, and $r_{\text {crit }}$ increases with increasing heliographic latitude $\lambda$. That is, at $r<r_{\text {crit }}, \Delta U_{\alpha \mathrm{p}}=U_{\mathrm{t} 2}$ and $Q_{\text {flow }}>0$. Then, at $r \geqslant r_{\text {crit }}, Q_{\text {flow }}=0$ and the deceleration of the alpha particles is governed by the rotational force.

\subsection{Method of Solution}

There are four principal unknowns in our model: $U_{\mathrm{p} r}, U_{\mathrm{p} \phi}$, $\Delta U_{\alpha \mathrm{p}}$, and $\psi_{B}$. To solve for these unknowns, we use the following four equations: Equations (4), (22), (23), and either Equation (28) or Equation (44). We choose between

\footnotetext{
5 Similar bounded-state models have been used to describe the local value of the plasma temperature anisotropy in space plasmas (cf Denton et al. 1994; Hellinger \& Trávníček 2008; Samsonov \& Pudovkin 2000; Samsonov et al. 2007).
}

Equations (28) and (44) based on the criteria set forth in Section 3.1.3. In practice, this works out as follows. Motivated by observations of the fast solar wind, we set $\Delta U_{\alpha \mathrm{p}}=U_{\mathrm{t} 2}$ at the innermost radius of our numerical solutions. This condition is just Equation (28). As we integrate outward from this innermost radius, we continue to use Equation (28) as long as Equation (45) is satisfied (which is the condition that the rotational force on its own would be unable to decelerate alpha particles to a drift velocity below the instability threshold). However, beyond a certain radius (denoted $r_{\text {crit }}$ ), Equation (45) is violated and the rotational force decelerates alpha particles to drift velocities smaller than $U_{\mathrm{t} 2}$. At $r>r_{\text {crit }}$, alpha-particle deceleration is controlled by the rotational force, and we use Equation (44) instead of Equation (28) as the fourth equation in our model. Numerically, we solve our model equations using a combined Euler and secant method (Press et al. 1992, 347ff).

When solving these four equations, we determine $\rho_{\alpha}$ and $\rho_{\mathrm{p}}$ using Equation (20), where we specify the constants $\mathcal{G}_{\mathrm{p}}$ and $\mathcal{G}_{\alpha}$ so as to match observations at the inner boundary. In addition, we determine $U_{\mathrm{t} 2}$ empirically, using analytic fits to the observed profiles of the magnetic field strength, $T_{\perp \alpha}$, and $T_{\| \alpha}$. As described further in Sections 4 and 5, we use different analytic fits for modeling the ecliptic plane at $r<1 \mathrm{AU}$ and nonzero heliographic latitudes at $r>1.5 \mathrm{AU}$.

We choose to estimate $v_{\mathrm{A}}$ empirically from observed magnetic field strengths rather than from the strength of the spiral magnetic field in our model because the magnetic field strength in our model omits the contribution from magnetic fluctuations. Magnetic fluctuations at scales comparable to the turbulence outer scale $L_{\mathrm{c}}$ (roughly $10^{6} \mathrm{~km}$ at $r=1 \mathrm{AU}$ ) are comparable in magnitude to the background magnetic field in the regions that we are interested in. FM/W instabilities are most unstable at very small wavelengths, comparable to the ion inertial length, which is $\ll L_{\mathrm{c}}$. For instabilities at these small wavelengths, the magnetic fluctuations at scales $\sim L_{\mathrm{c}}$ appear like a uniform field. It is thus the total magnetic field strength, including these large-scale magnetic fluctuations, that is relevant for determining the instability thresholds.

\section{NUMERICAL SOLUTION FOR THE INNER HELIOSPHERE AT ZERO HELIOGRAPHIC LATITUDE}

In this section, we choose the innermost radius of our numerical solution, denoted $r_{0}$, to be $r_{0}=0.29 \mathrm{AU}$, which is the perihelion of the Helios satellite mission. To determine the proton number density $n_{\mathrm{p}}=\rho_{\mathrm{p}} / m_{\mathrm{p}}$ at $r=r_{0}$, we average the measured values of $n_{\mathrm{p}}=33.2 \mathrm{~cm}^{-3}, n_{\mathrm{p}}=28.3 \mathrm{~cm}^{-3}$, and $n_{\mathrm{p}}=$ $29.4 \mathrm{~cm}^{-3}$ at $r \simeq r_{0}$ in the fast solar wind reported by Marsch et al. (1982c) and Bourouaine \& Chandran (2013). This gives $n_{\mathrm{p}}=30.3 \mathrm{~cm}^{-3}$ at $r=r_{0}$. We set $U_{\mathrm{p} r}\left(r_{0}\right)=700 \mathrm{~km} \mathrm{~s}^{-1}$ as a characteristic fast-solar-wind speed. We then set $\rho_{\alpha}\left(r_{0}\right)=0.2 \rho_{\mathrm{p}}\left(r_{0}\right)$. These boundary values at $r=r_{0}$ allow us to evaluate the constant $\mu$ in Equation (13). We note that, upon integrating the equations of our model, we obtain $n_{\mathrm{p}}=2.5 \mathrm{~cm}^{-3}$ at $r=1 \mathrm{AU}$, which is close to the observed average value of $n_{\mathrm{p}}=2.7 \mathrm{~cm}^{-1}$ in the fast solar wind measured by Ulysses, scaled to $r=1 \mathrm{AU}$ (McComas et al. 2000). We set $r_{\mathrm{eff}}=10 R_{\odot}$ and integrate from $r=r_{0}$ to $r=1$ AU using 3000 radial grid points. For the total magnetic field strength, we adopt the radial profile obtained from fits to Helios measurements in fast-wind 
streams (Mariani et al. 1979)

$$
B(r)=3.28 \times 10^{-5} \mathrm{G}\left(\frac{r}{1 \mathrm{AU}}\right)^{-1.86}, \quad \text { for } \quad r<1 \mathrm{AU}
$$

We use Equation (49) to determine $v_{\mathrm{A}}$.

When evaluating $U_{\mathrm{t} 2}$, we treat $T_{\perp \alpha}(r)$ and $T_{\| \alpha}(r)$ as known functions of the radius. To determine these functions, we make use of results from Marsch et al. (1982b), who fit Helios measurements of $T_{\perp \alpha}(r)$ and $T_{\| \alpha}(r)$ to power laws in $r$ for solarwind streams with $600 \mathrm{~km} \mathrm{~s}^{-1}<U_{\mathrm{pr}}<700 \mathrm{~km} \mathrm{~s}^{-1}$ and for solarwind streams with $700 \mathrm{~km} \mathrm{~s}^{-1}<U_{\mathrm{p} r}<800 \mathrm{~km} \mathrm{~s}^{-1}$. To obtain power-law fits for $T_{\perp \alpha}(r)$ and $T_{\| \alpha}(r)$ for solar-wind streams with $U_{\mathrm{p} r} \simeq 700 \mathrm{~km} \mathrm{~s}^{-1}$, we average the power law indices obtained by Marsch et al. (1982b) for these two wind-speed ranges. We then normalize the $T_{\perp \alpha}$ power law so that $T_{\perp \alpha}(1 \mathrm{AU})$ matches the average of the values of $T_{\perp \alpha}$ at $r=1 \mathrm{AU}$ found by Marsch et al. (1982b) for these two wind-speed ranges, and likewise for $T_{\| \alpha}$. This gives

$$
T_{\perp \alpha}=7 \times 10^{5} \mathrm{~K}\left(\frac{r}{1 \mathrm{AU}}\right)^{-1.37}
$$

and

$$
T_{\| \alpha}=8 \times 10^{5} \mathrm{~K}\left(\frac{r}{1 \mathrm{AU}}\right)^{-1.155} .
$$

Variations in the assumed temperature profiles lead to significantly different results in our model, as we discuss further in the Appendix. For reference, we plot the radial profile of

$$
\beta_{\| \mathrm{p}} \equiv \frac{8 \pi n_{\mathrm{p}} k_{\mathrm{B}} T_{\| \mathrm{p}}}{B^{2}}
$$

in Figure 4 that results in our numerical solution, where $T_{\| \mathrm{p}}$ is the parallel proton temperature, which we evaluate using Helios observations (Equation (58) below).

\subsection{Proton and Alpha-particle Velocities}

At all radii explored in this section (0.29-1 AU), $r<r_{\text {crit }}$, and thus $\Delta U_{\alpha \mathrm{p}}=U_{\mathrm{t} 2}$ in our model. We show the radial profiles of $v_{\mathrm{A}}, U_{\mathrm{t} 1}, \Delta U_{\alpha \mathrm{p}}=U_{\mathrm{t} 2}$, and $U_{\mathrm{p} r}$ in our model in Figure 2, along with Helios measurements of $\Delta U_{\alpha \mathrm{p}}$ from Marsch et al. (1982b). We note that in our model the radial proton velocity $U_{\mathrm{p} r}$ increases by about $4 \%$ between $0.29 \mathrm{AU}$ and $1 \mathrm{AU}$ to conserve momentum as the alpha particles decelerate.

The instability threshold $U_{\mathrm{t} 1}$ for the A/IC instability is smaller than the threshold $U_{\mathrm{t} 2}$ for the FM/W instability at $r \lesssim 0.65 \mathrm{AU}$ in our model. Nevertheless, the observed drift speed from Marsch et al. (1982b) follows our profile for the FM/W instability threshold (i.e., $\left.U_{\mathrm{t} 2}\right)$ very well, even in the range in which $U_{\mathrm{t} 1}<U_{\mathrm{t} 2}$. This finding supports our assumption that it is the FM/W instability and not the A/IC instability that limits $\Delta U_{\alpha \mathrm{p}}$ in the solar wind. However, as we discuss further in the Appendix, this finding is sensitive to variations in the assumed profiles of $T_{\perp \alpha}$ and $T_{\| \alpha}$.

We show the radial profiles of the azimuthal velocity components $U_{\mathrm{p} \phi}$ and $U_{\alpha \phi}$ in Figure 3. While $U_{\mathrm{p} \phi}$ is positive, $U_{\alpha \phi}$ is negative, and both velocities decrease slowly (more slowly than $1 / r)$ with increasing $r$. In addition, we show the solution for $U_{\mathrm{p} \phi}$ without alpha particles (i.e., Equation (22) without the last term on the right-hand side). The azimuthal component of the velocity decreases $\propto r^{-1}$ in this case. At the effective corotation radius $r_{\text {eff }}$, we have taken both particle species to have the same azimuthal velocity. Due to the bending of the magnetic field lines, however, the azimuthal velocity of the alpha particles changes sign at some point between $r_{\text {eff }}$ and $r_{0}$. These results for the azimuthal flow are in agreement with previous studies of angular-momentum transport in the solar wind and show the importance of the differential streaming for the azimuthal-flow components and angular-momentum transport in the solar wind ( $\mathrm{Li} \& \mathrm{Li}$ 2006; Li et al. 2007). Our model, however, extends these previous treatments by including the interplay of micro-instabilities and the rotational force.

\subsection{The Parker Spiral Field}

The classic description of the interplanetary magnetic field was given by Parker (1958). The angle $\psi_{B}$ between $\widehat{\boldsymbol{r}}$ and $\boldsymbol{B}$ in the Parker model (with our sign convention) is given by

$$
\tan \psi_{B}=\frac{B_{\phi}}{B_{r}}=\frac{\Omega_{\odot} \sin \theta}{U_{\mathrm{p} r}}\left(r_{\mathrm{eff}}-r\right) .
$$

Parker's model neglects alpha particles and assumes that $U_{\mathrm{p} r}$ and $U_{\mathrm{p} \phi}$ are independent of $r$ in a non-rotating reference frame. Therefore, the specific angular momentum of the solar wind increases with distance from the Sun in his model, which implies an ongoing torque on the plasma. In our model, the total torque on the solar-wind fluid is zero beyond the effective co-rotation radius. In a self-consistent solution of the momentum and induction equations in single-fluid MHD, Weber \& Davis (1967) found a solution that is in some sense intermediate between Parker's and ours, in that the tangential flow velocity decreases with $r$, but not as rapidly as $r^{-1}$ because of the Lorentz force. While our solution assumes zero total torque, the interaction between protons and alpha particles still leads to torques that act on the ion species individually.

We compare our torque-free solution for $\psi_{B}$ with Parker's solution in Figure 4. As this figure shows, our value for $\psi_{B}$ is very similar to Parker's but slightly larger. The reason for this is that $U_{\mathrm{p} \phi}$ is smaller in our model than in Parker's (which can be seen in Figure 3, upon noting that $U_{\mathrm{p} \phi}=20.5 \mathrm{~km} \mathrm{~s}^{-1}$ in Parker's model at all radii given that we have set $\left.r_{\mathrm{eff}}=10 R_{\odot}\right)$. The smaller $\phi$ velocities in our model cause the field lines to "bend back" in the $-\widehat{\phi}$ direction to a greater degree than in Parker's model. This difference is accentuated if we set $\rho_{\alpha}=0$ in our model, which leads to an even larger reduction in $U_{\mathrm{p} \phi}$ (which, again, is shown in Figure 3).

\subsection{Heating from Alpha-particle Deceleration}

In Figure 5, we plot the value of $Q_{\text {flow }}$ in our model solution for the inner heliosphere in the heliographic equator. The radial profile of $Q_{\text {flow }}$ for $r_{0}<r<1 \mathrm{AU}$ is well-fit by a power law of the form

$$
Q_{\text {flow }} \approx 4.1 \times 10^{-4} \mathrm{erg} \mathrm{cm}^{-3} \mathrm{~s}^{-1}\left(\frac{r}{R_{\odot}}\right)^{-5.47} .
$$

The "empirical" perpendicular and parallel heating rates $Q_{\perp j}$ and $Q_{\| j}$ required to explain the observed temperature profiles of 


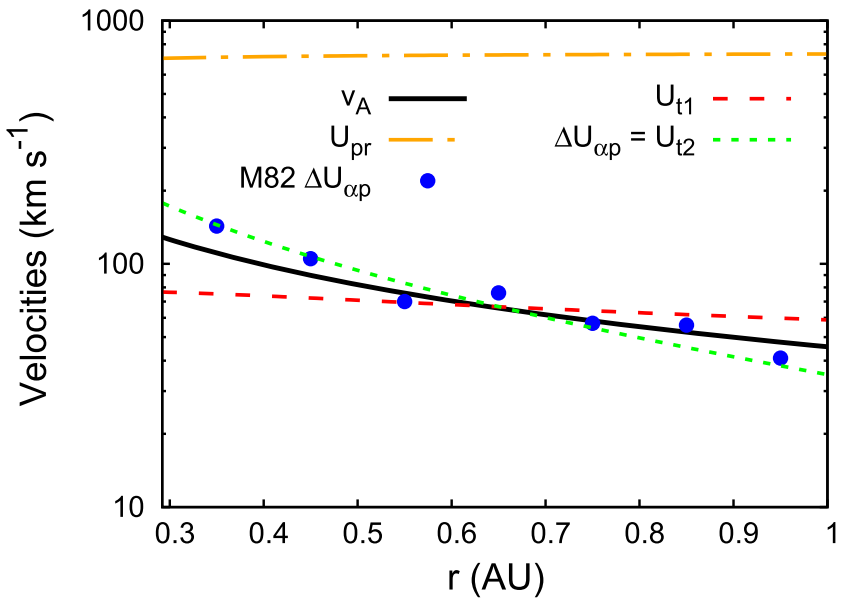

Figure 2. Radial profiles of $v_{\mathrm{A}}, U_{\mathrm{t} 1}, \Delta U_{\alpha \mathrm{p}}=U_{\mathrm{t} 2}$, and $U_{\mathrm{p} r}$ in our model in the heliographic equatorial plane. The points "M82 $\Delta U_{\alpha \mathrm{p}}$ " represent the Helios measurements in fast-solar-wind streams reported by Marsch et al. (1982b).

protons $(j=\mathrm{p})$ and alpha particles $(j=\alpha)$ in the solar wind are given by (Chandran et al. 2011; Chew et al. 1956; Sharma et al. 2006)

$$
Q_{\perp j}=B n_{j} k_{\mathrm{B}} U_{j r} \frac{\partial}{\partial r}\left(\frac{T_{\perp j}}{B}\right)
$$

and

$$
Q_{\| j}=\frac{n_{j}^{3} k_{\mathrm{B}} U_{j r}}{2 B^{2}} \frac{\partial}{\partial r}\left(\frac{B^{2} T_{\| j}}{n_{j}^{2}}\right) .
$$

To evaluate these empirical heating rates, we determine $B$ using Equation (49), and we set $n_{j}$ equal to the value in our solar-wind model for the inner heliosphere. To evaluate $T_{\perp \alpha}$ and $T_{\| \alpha}$, we use Equations (50) and (51). To determine $T_{\perp \mathrm{p}}$ and $T_{\| \mathrm{p}}$, we average the fits from Marsch et al. (1982c) to the proton-temperature profiles in fast-wind streams with $600 \mathrm{~km} \mathrm{~s}^{-1}<U_{\mathrm{p} r}<$ $700 \mathrm{~km} \mathrm{~s}^{-1}$ and $700 \mathrm{~km} \mathrm{~s}^{-1}<U_{\mathrm{pr}}<800 \mathrm{~km} \mathrm{~s}^{-1}$, which leads to

$$
T_{\perp \mathrm{p}}=2 \times 10^{5} \mathrm{~K}\left(\frac{r}{1 \mathrm{AU}}\right)^{-1.125}
$$

and

$$
T_{\| \mathrm{p}}=2 \times 10^{5} \mathrm{~K}\left(\frac{r}{1 \mathrm{AU}}\right)^{-0.72} .
$$

We plot the empirical heating rates determined in this way in Figure 5. The values of $Q_{\| \mathrm{p}}$ and $Q_{\| \alpha}$ given by Equation (55) are both negative (cf Hellinger et al. 2011; Hellinger \& Trávníček 2013), but we plot their absolute values.

As Figure 5 shows, $Q_{\text {flow }}$ exceeds the empirical heating rate $Q_{\perp \alpha}$ at $0.29 \mathrm{AU}<r \lesssim 1 \mathrm{AU}$. At $r<0.42 \mathrm{AU}, Q_{\text {flow }} \simeq Q_{\perp \mathrm{p}}$. The ratio $Q_{\text {flow }} / Q_{\perp \mathrm{p}}$ decreases as $r$ increases, reaching a value of $1 / 4$ at $r=1$ AU. We conclude that alpha-particle deceleration makes an important contribution to the heating of the fast solar wind at $0.29 \mathrm{AU}<r<1 \mathrm{AU}$. In addition, the fact that $Q_{\text {flow }} / Q_{\perp \mathrm{p}}$ increases from $\simeq 1 / 4$ to $\simeq 1$ as $r$ decreases from $1 \mathrm{AU}$ to $0.29 \mathrm{AU}$ suggests that alpha-particle deceleration plays an important role in the energetics of the solar wind at $r<0.29$ AU.

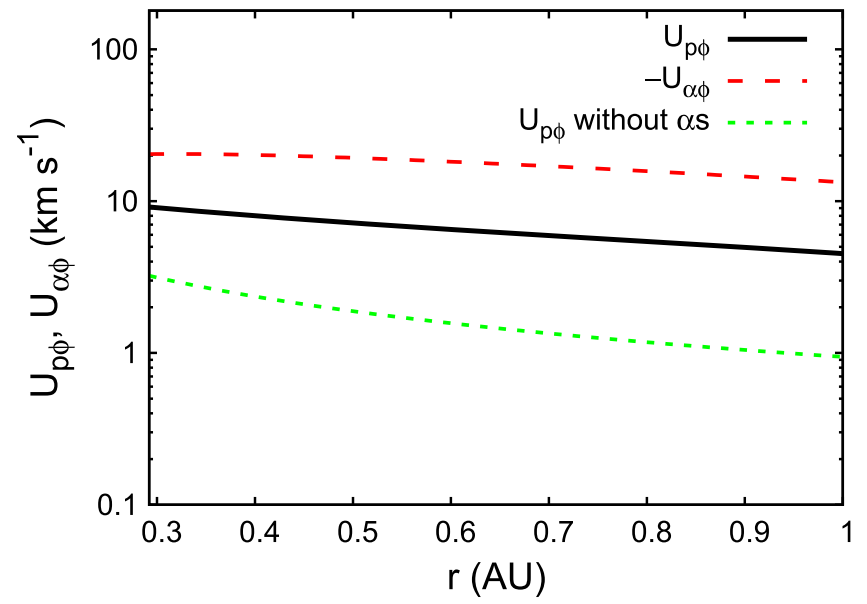

Figure 3. Radial profiles of the azimuthal velocities $U_{\mathrm{p} \phi}$ and $U_{\alpha \phi}$ in our model in the heliographic equatorial plane. We also show the profile of the azimuthal velocity in our model in the limit $\rho_{\alpha} \rightarrow 0$.

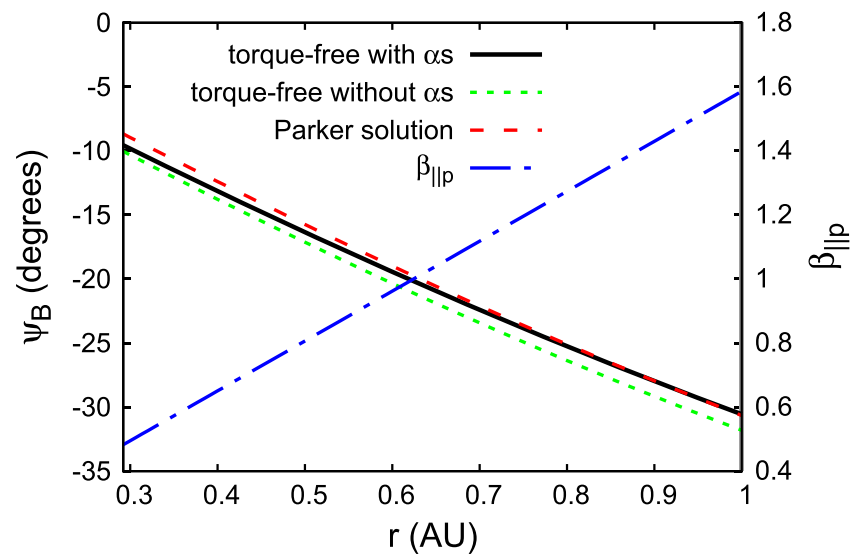

Figure 4. Angle $\psi_{B}$ between $\widehat{\boldsymbol{r}}$ and $\boldsymbol{B}$ as a function of heliocentric distance $r$ in the heliographic equatorial plane. We show our torque-free model (Equation (23)), a torque-free model without alpha particles, and Parker's model (Equation (53)), which corresponds to $U_{\mathrm{p} \phi}=$ constant. The axis on the righthand side provides the scale for the plot (dashed-dotted blue line) of $\beta_{\| \mathrm{p}}$ (Equation (52)).

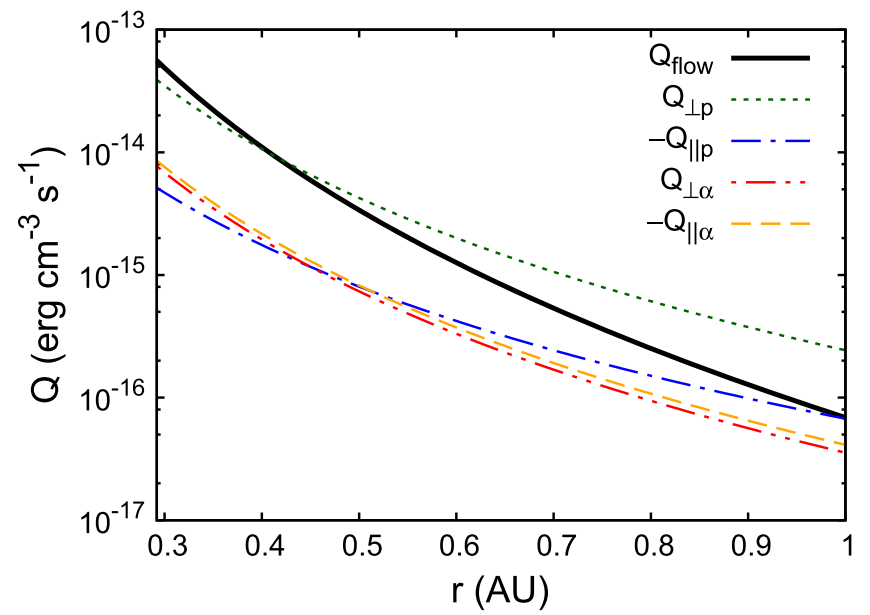

Figure 5. Comparison of $Q_{\text {flow }}$ at zero heliographic latitude with the "empirical" heating rates $Q_{\perp \mathrm{p}}, Q_{\| \mathrm{p}}, Q_{\perp \alpha}$, and $Q_{\| \alpha}$ required to explain the observed profiles of, respectively, $T_{\perp \mathrm{p}}, T_{\| \mathrm{p}}, T_{\perp \alpha}$, and $T_{\| \alpha}$ (Equations (55) and (56)). The parallel heating rates $Q_{\| \mathrm{p}}$ and $Q_{\| \alpha}$ are negative (cf Hellinger \& Trávníček 2013), but we have plotted their absolute values. 


\section{NUMERICAL SOLUTIONS FOR THE OUTER HELIOSPHERE AT NONZERO HELIOGRAPHIC LATITUDE}

In this section, we present model solutions for the fast solar wind at heliocentric distances between 1.5 and $4.2 \mathrm{AU}$ for a range of heliographic latitudes. We then compare our results with the Ulysses measurements reported by Reisenfeld et al. (2001), which were taken during the outbound leg of Ulysses's first orbit. As in Section 4, there are several quantities that we need to specify in order to solve for the radial profiles of $U_{\mathrm{p} r}, U_{\mathrm{p} \phi}$, $\Delta U_{\alpha \mathrm{p}}$, and $\psi_{B}$ (from which we can then determine $U_{\alpha r}$ and $U_{\alpha \phi}$ using Equations (9) and (10)). We set the innermost radius in these solutions, denoted $r_{0, \mathrm{U}}$, to be $1.5 \mathrm{AU}$. We set $n_{\mathrm{p}}\left(r_{0, \mathrm{U}}\right)=1.2 \mathrm{~cm}^{-3}, n_{\alpha}\left(r_{0, \mathrm{U}}\right)=0.05 n_{\mathrm{p}}\left(r_{0, \mathrm{U}}\right)$, and $U_{\mathrm{p} r}\left(r_{0, \mathrm{U}}\right)=$ $758 \mathrm{~km} \mathrm{~s}^{-1}$, in agreement with Ulysses observations (McComas et al. 2000). In order to match the magnetic field strength seen in the Reisenfeld et al. (2001) observations, we fit the $v_{\mathrm{A}}$ measurements of Reisenfeld et al. (2001) to a power law of the form

$$
v_{\mathrm{A}}=64.7 \mathrm{~km} \mathrm{~s}^{-1}\left(\frac{r}{1 \mathrm{AU}}\right)^{-0.49}, \quad \text { for } \quad r>1.5 \mathrm{AU},
$$

and we assume that this same power law holds at all values of $\theta$. We then determine $B$ using Equations (59), (1), and the proton density in our numerical solutions. To determine $U_{\mathrm{t} 2}$ in Equation (25), we adopt the total-alpha-particle temperature profile inferred by McComas et al. (2000) from Ulysses observations:

$$
\begin{aligned}
T_{\alpha} & =\frac{2 T_{\perp \alpha}+T_{\| \alpha}}{3} \\
& =\left[1.42 \times 10^{6} \mathrm{~K}-(871 \mathrm{~K}) \lambda\right]\left(\frac{r}{1 \mathrm{AU}}\right)^{-0.8},
\end{aligned}
$$

where $\lambda=90^{\circ}-\theta$ is the heliographic latitude in degrees. Reisenfeld et al. (2001) found that $T_{\perp \alpha} / T_{\| \alpha}=0.87 \pm 0.092$ over their entire data set, covering the radial range $1.5 \mathrm{AU}<r<4.2 \mathrm{AU}$. For our fiducial model, we thus set

$$
\frac{T_{\perp \alpha}}{T_{\| \alpha}}=0.87
$$

With the above boundary conditions and profiles for $T_{\perp \alpha}$, $T_{\| \alpha}$, and $v_{\mathrm{A}}$, we integrate the equations of our model from $r_{0, \mathrm{U}}=1.5 \mathrm{AU}$ out to $4.2 \mathrm{AU}$ at 1500 different values of the heliographic latitude $\lambda$. For each value of $\lambda$, we use a grid of $\simeq 3000$ points in the $r$ direction. To connect our results to Ulysses observations, we use the Ulysses orbital elements from Balogh et al. (2001, p. 18) to map heliocentric distance $r$ to heliographic latitude $\lambda$ along the portion of the Ulysses trajectory considered by Reisenfeld et al. (2001). This mapping results in either a multi-valued function $r_{\text {Ulysses }}(\lambda)$ or a singlevalued function $\lambda(r)$ and is plotted as the dashed line in Figure 6. We also plot in this figure the value of $r_{\text {crit }}$ as a function of $\lambda$ in our numerical solutions. The two curves $r_{\text {crit }}(\lambda)$ and $r_{\text {Ulysses }}(\lambda)$ intersect at $r \approx 3.3 \mathrm{AU}$. Thus, when Ulysses was at $r<3.3 \mathrm{AU}$, alpha particles were decelerated by instabilities at the spacecraft location. In contrast, at $r>3.3 \mathrm{AU}$, the local deceleration of alpha particles at the spacecraft location resulted from the rotational force.

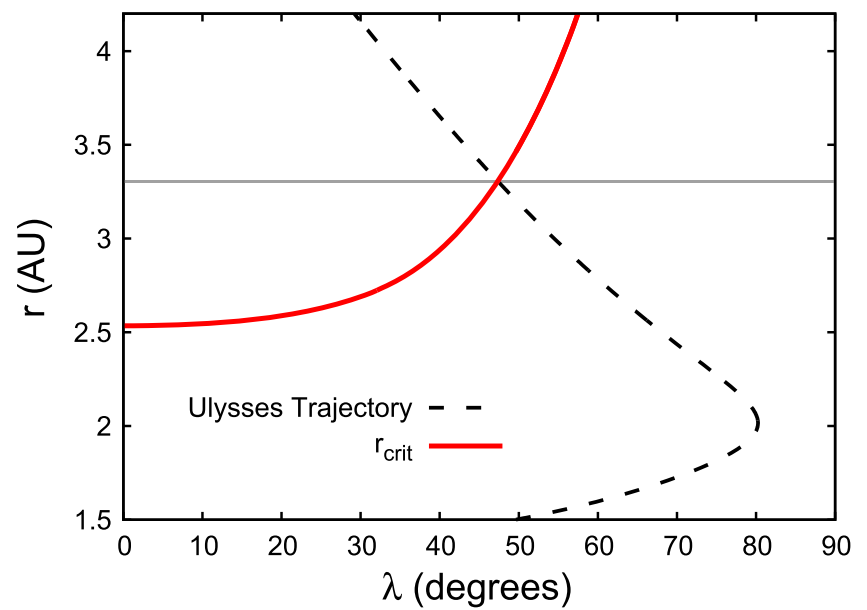

Figure 6. Heliocentric distance of the Ulysses spacecraft as a function of the spacecraft's heliographic latitude $\lambda$ during the outbound leg of its first polar orbit (black dashed line). The red solid line shows the value of $r_{\text {crit }}$ as a function of $\lambda$ in our model (based on Equation (62)). The horizontal line marks the heliocentric distance $r=3.3$ AU beyond which Ulysses was outside the critical radius $r_{\text {crit }}(\lambda)$.

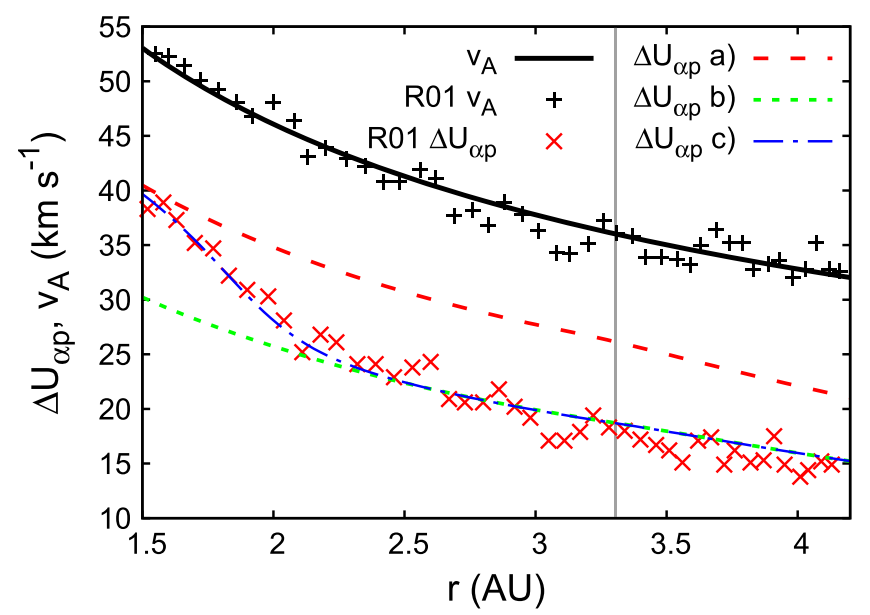

Figure 7. Radial profiles of $v_{\mathrm{A}}$ and $\Delta U_{\alpha \mathrm{p}}$ in our model along the trajectory of the Ulysses spacecraft during the outbound leg of its first polar orbit. We use the following temperature anisotropies: (a) $T_{\perp \alpha} / T_{\| \alpha}=0.87 ; \quad$ (b) $T_{\perp \alpha} / T_{\| \alpha}=0.80$; and (c) Equation (62). The points "R01" show observations from Reisenfeld et al. (2001). The vertical line shows the position of $r_{\text {crit }}(\lambda)$.

In Figure 7, we plot the drift speed in our numerical solutions along the Ulysses orbit, $\Delta U_{\alpha \mathrm{p}}(r, \lambda(r))$. We also plot the Alfvén speed from Equation (59), as well as the observed values of $v_{\mathrm{A}}$ and $\Delta U_{\alpha \mathrm{p}}$ from Reisenfeld et al. (2001). By setting $T_{\perp \alpha} / T_{\| \alpha}=0.87$, we obtain solutions for $\Delta U_{\alpha p}$ that are in good agreement with the observations at $r \simeq 1.5 \mathrm{AU}$, but in poor agreement at larger $r$. On the other hand, if we replace Equation (61) with $T_{\perp \alpha} / T_{\| \alpha}=0.80$ and repeat our numerical calculations at all 1500 values of $\lambda$, then we obtain the drift speed plotted as a green dashed line in Figure 7, which agrees well with the measured value of $\Delta U_{\alpha \mathrm{p}}$ at $r>2 \mathrm{AU}$. We are in fact able to reproduce the observed value of $\Delta U_{\alpha \mathrm{p}}$ over the entire radial range of $1.5 \mathrm{AU}<r<4.2 \mathrm{AU}$ by taking $T_{\perp \alpha} / T_{\| \alpha}$ to transition smoothly from the value 0.87 at $r=1.5 \mathrm{AU}$ to the value 0.80 at $r>2 \mathrm{AU}$. To show this, we compute a third family of numerical solutions at all 1500 values of $\lambda$ in which we replace Equation (61) with the temperature-anisotropy 
profile

$$
\frac{T_{\perp \alpha}}{T_{\| \alpha}}=0.87-0.035\left[\tanh \left(3.5\left(\frac{r}{1 \mathrm{AU}}-1.85\right)\right)+1\right] .
$$

Although the temperature-anisotropy profile in Equation (62) enables our model to reproduce the observed $\Delta U_{\alpha \mathrm{p}}$ profile, we are aware of no reason that the temperature-anisotropy profile should follow this particular form. Thus, all we can conclude is that, given the observational uncertainty in the alpha-particle temperature anisotropy, our model could be consistent with the $\Delta U_{\alpha \mathrm{p}}$ measurements. However, it could equally well be inconsistent with the $\Delta U_{\alpha \mathrm{p}}$ measurements if the true alphaparticle temperature anisotropy deviates sufficiently from the form in Equation (62).

As discussed above, the FM/W instability is responsible for the alpha-particle deceleration seen in Figure 7 at $r<3.3 \mathrm{AU}$. The drift speed at these heliocentric distances is significantly smaller than $v_{\mathrm{A}}$, because $T_{\perp \alpha}<T_{\| \alpha}$ and reducing $T_{\perp \alpha} / T_{\| \alpha}$ lowers the minimum drift speed needed to excite the FM/W instability. At $r>3.3 \mathrm{AU}$, instabilities no longer contribute to the deceleration of the alpha particles. However, the rotational force continues to decelerate the alpha particles, leading to a good agreement between the observations and two of the three families of solutions that we have computed (the solutions in which Equation (61) is replaced by either $T_{\perp \alpha} / T_{\| \alpha}=0.80$ or Equation (62)).

Reisenfeld et al. (2001) also calculated the values of $\Delta U_{\alpha p}$ that result from alpha-particle deceleration by the rotational force. For this calculation, these authors took the rotational force to be the dominant deceleration mechanism throughout the radial interval 1.5 AU $<r<4.2 \mathrm{AU}$. The values we obtain for $\Delta U_{\alpha p}$ are much smaller than the values obtained by Reisenfeld et al. (2001), because in our model instabilities control the deceleration at 1.5 $\mathrm{AU}<r<3.3 \mathrm{AU}$, a region in which instabilities are more effective than the rotational force at decelerating alpha particles. Then, when the rotational force takes over in our model at $r=3.3 \mathrm{AU}$, the alpha particles are already at a much smaller drift speed than in Reisenfeld et al.'s (2001) calculation.

In Figure 8, we plot the energy release rate $Q_{\text {flow }}(r, \lambda(r))$ in our model (using Equation (62)) and the empirical proton and alpha-particle heating rates given in Equations (55) and (56) evaluated along the Ulysses trajectory. We evaluate the radial derivatives of $n_{\alpha}, n_{\mathrm{p}}$, and $B$ in Equations (55) and (56) using our model solutions (based on Equation (62)), and we determine $T_{\perp \alpha}$ and $T_{\| \alpha}$ using Equations (60) and (62). We take $T_{\perp \mathrm{p}}=T_{\| \mathrm{p}}=T_{\mathrm{p}}$, where

$$
T_{\mathrm{p}}=\left[2.58 \times 10^{5} \mathrm{~K}+(223 \mathrm{~K}) \lambda\right]\left(\frac{r}{1 \mathrm{AU}}\right)^{-1.02}
$$

is the proton temperature observed by Ulysses as reported by McComas et al. (2000), and $\lambda=90^{\circ}-\theta$ is the heliographic latitude, which in Equation (63) is expressed in degrees. Matteini et al. (2013) report a weak temperature anisotropy with $T_{\perp \mathrm{p}}<T_{\| \mathrm{p}}$ for the total proton distribution. However, the proton-core and the proton-beam populations exhibit opposite anisotropies. For the sake of simplicity, we assume that the proton distribution is a single and isotropic plasma component.
As Figure 8 shows, $Q_{\text {flow }} \simeq Q_{\perp \alpha}$ at $r \simeq 1.8 \mathrm{AU}$, and $Q_{\text {flow }}$ is a substantial fraction of the alpha-particle heating rate at $r \lesssim 2.2$ AU. However, at larger radii, $Q_{\text {flow }} / Q_{\perp \alpha}$ decreases to small values, and at $r>3.3 \mathrm{AU}, Q_{\text {flow }}=0$, since the alphaparticle deceleration at these radii is governed by the rotational force.

We note that if we were to set $n_{\mathrm{p}} \propto r^{-2}$, then Equations (59) and (63) and the condition $T_{\| \mathrm{p}}=T_{\mathrm{p}}$ imply that $B^{2} T_{\| \mathrm{p}} / n_{\mathrm{p}}^{2} \propto r^{0}$, which leads to $Q_{\| \mathrm{p}}=0$ in Equation (56). This means that $Q_{\| \mathrm{p}}$ in Figure 8 is nonzero only because of the deviation of $n_{\mathrm{p}}$ from an $r^{-2}$ profile. The reason that $Q_{\| \mathrm{p}} \ll Q_{\perp \mathrm{p}}$ in Figure 8 is that $n_{\mathrm{p}}$ is close to an $r^{-2}$ profile. The fact that $Q_{\| \mathrm{p}} \ll Q_{\perp \mathrm{p}}$ along the Ulysses orbit given the observed profiles of $B, n_{\mathrm{p}}$, and $T_{\mathrm{p}}$ suggests that turbulent heating results in the inequality $Q_{\| \mathrm{p}} \ll Q_{\perp \mathrm{p}}$ in the solar wind. This inequality was also obtained in the solar-wind model developed by Chandran et al. (2011), which included an analytic model of plasma heating by low-frequency solar-wind turbulence, in which the turbulence dissipates via Landau damping, transit-time damping, and stochastic heating.

\section{THE COASTING APPROXIMATION}

In Equation (4), we assume that the net force on the plasma is negligible. We call this the coasting approximation. In this section, we discuss the applicability of this approximation to the solar wind. Since $U_{\mathrm{p} r}$ and $U_{\alpha r}$ asymptote toward constant values at large $r$, we expect that the most stringent test for the coasting approximation occurs at the smallest heliocentric distances that we consider. We thus focus in this section on the region

$$
0.29 \mathrm{AU}<r<1 \mathrm{AU}
$$

in which alpha-particle deceleration is controlled by instabilities.

To estimate the sizes of different forces, we make the simplifying approximations that, when Equation (64) is satisfied, $B \propto n_{\mathrm{p}} \propto n_{\alpha} \propto r^{-2}$, which implies that $v_{\mathrm{A}} \propto r^{-1}$. Since $U_{\mathrm{p} r}$ is only weakly dependent on $r$ in this range of heliocentric distances, the alpha particles experience an acceleration of $\simeq U_{\alpha r}(\partial / \partial r) \Delta U_{\alpha \mathrm{p}}$, which is also, very roughly, $\simeq U_{\mathrm{p} r}(\partial / \partial r) \Delta U_{\alpha \mathrm{p}}$. Since $\Delta U_{\alpha \mathrm{p}} \sim v_{\mathrm{A}}$ in this region, the net force per unit volume on the alpha particles needed to cause this deceleration is

$$
F_{\text {decl. }} \sim\left|\rho_{\alpha} U_{\mathrm{p} r} \frac{\partial v_{\mathrm{A}}}{\partial r}\right| \sim \rho_{\alpha 0} U_{\mathrm{p} r} \frac{v_{\mathrm{A} 0}}{r_{0}}\left(\frac{r}{r_{0}}\right)^{-4},
$$

where the subscript 0 indicates that a quantity is evaluated at $r=r_{0}=0.29 \mathrm{AU}$. Within the coasting approximation, the protons also experience a net force of magnitude $F_{\text {decl. }}$ as the alpha particles are decelerated, but the direction of this force is opposite to the direction of the force experienced by the alpha particles. We conjecture that the coasting approximation is valid if $F_{\text {decl. }}$ is substantially larger than the other forces experienced by alpha particles and protons. We now estimate these other forces. 


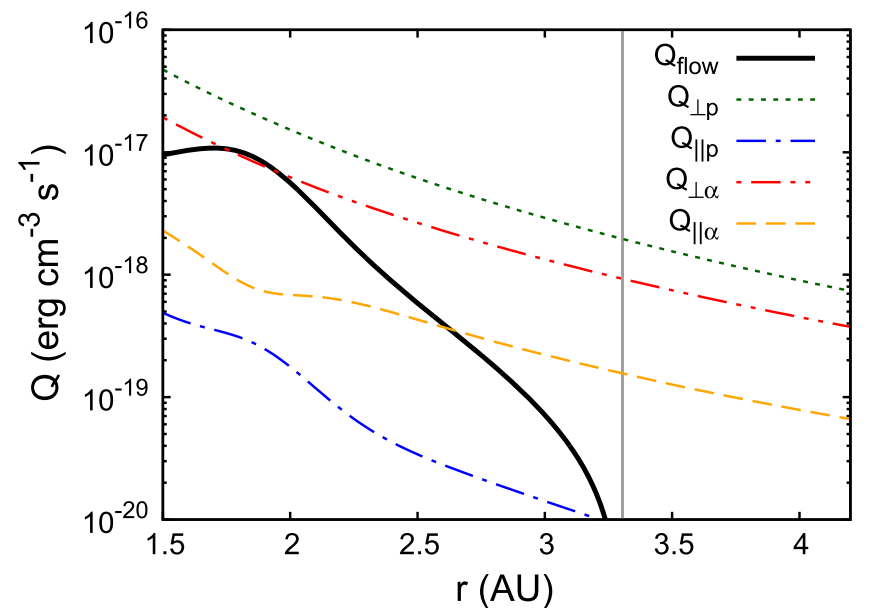

Figure 8. Energy-release rate $Q_{\text {flow }}$ and empirical heating rates $Q_{\perp \mathrm{p}}, Q_{\| \mathrm{p}}, Q_{\perp \alpha}$, and $Q_{\| \alpha}$ (Equations (55) and (56)) evaluated along the trajectory $(r, \lambda(r))$ of the Ulysses spacecraft. The vertical line shows the radius $r=3.3 \mathrm{AU}$ at which Ulysses crossed the critical radius $r_{\text {crit }}(\lambda)$ (see Figure 6).

The gravitational force per unit volume on the protons is given by

$$
F_{\mathrm{G}}=\frac{G M_{\odot} \rho_{\mathrm{p}}}{r^{2}} \sim \frac{G M_{\odot} \rho_{\mathrm{p} 0}}{r_{0}^{2}}\left(\frac{r}{r_{0}}\right)^{-4}
$$

where $G$ is the gravitational constant, and $M_{\odot}$ is the mass of the Sun. The gravitational force per unit volume on the alpha particles is smaller than $F_{\mathrm{G}}$ by a factor of $\rho_{\alpha} / \rho_{\mathrm{p}}$, and so we neglect it henceforth.

The wave pressure force on protons per unit volume exerted by Alfvén waves is given by

$$
F_{\mathrm{w}}=-\frac{1}{2} \frac{\partial \mathcal{E}_{\mathrm{w}}}{\partial r}
$$

where $\mathcal{E}_{\mathrm{w}}$ is the wave energy density (Dewar 1970). We assume that $\mathcal{E}_{\mathrm{w}}$ is dominated by outward-propagating Alfvén waves, so that

$$
\mathcal{E}_{\mathrm{w}}=\frac{\rho_{\mathrm{p}}\left(z_{\mathrm{rms}}^{+}\right)^{2}}{4}
$$

where $z_{\text {rms }}^{+}$is the root mean square value of the Elsasser variable $z^{+} \equiv \delta \boldsymbol{v}-\delta \boldsymbol{B} / \sqrt{4 \pi \rho_{\mathrm{p}}}$ (Dewar 1970). Chandran \& Hollweg (2009) developed an analytical model for reflectiondriven Alfvén-wave turbulence in the solar wind. They found that

$$
z_{\mathrm{rms}}^{+}=z_{\mathrm{rms}, \mathrm{A}}^{+}\left(\frac{2 \eta^{1 / 4}}{1+\eta^{1 / 2}}\right)\left(\frac{v_{\mathrm{A}}}{v_{\mathrm{A}, \mathrm{A}}}\right)^{1 / 2}
$$

where $\eta \equiv \rho_{\mathrm{p}} / \rho_{\mathrm{p}, \mathrm{A}}$, and $\rho_{\mathrm{p}, \mathrm{A}}$ and $z_{\text {rms,A }}^{+}$are the values of $\rho_{\mathrm{p}}$ and $z_{\text {rms }}^{+}$at the Alfvén critical radius $r=r_{\mathrm{A}}$. With these quantities, we estimate the wave pressure force density as

$$
F_{\mathrm{w}} \sim \frac{2 \rho_{\mathrm{p} 0}}{r_{0}}\left(z_{\mathrm{rms}, \mathrm{A}}^{+}\right)^{2}\left(\frac{r_{\mathrm{A}}}{r_{0}}\right)^{2}\left(\frac{r}{r_{0}}\right)^{-5} .
$$

The rms amplitudes of turbulent velocity fluctuations in the fast solar wind are similar to the proton thermal velocities (Marsch et al. 1982c; Marsch 1986; Tu \& Marsch 1995). As a consequence, $\mathcal{E}_{\mathrm{w}}$ is similar to the plasma pressure $p$, and $|\nabla p| \sim p / r$. Thus, the plasma pressure force is small compared to $F_{\text {decl. }}$ if $F_{\mathrm{w}}$ is small compared to $F_{\text {decl. }}$.

Equations (65) and (66) yield

$$
\left|\frac{F_{\mathrm{G}}}{F_{\text {decl. }}}\right| \sim \frac{\rho_{\mathrm{p} 0} G M_{\odot}}{\rho_{\alpha 0} U_{\mathrm{p} r} v_{\mathrm{A} 0} r_{0}},
$$

which is 0.17 for $r_{0}=0.29 \mathrm{AU}, v_{\mathrm{A} 0} \approx 130 \mathrm{~km} \mathrm{~s}^{-1}$, and $\rho_{\alpha 0}=0.2 \rho_{\mathrm{p} 0}$. Equations (65) and (70) yield

$$
\left|\frac{F_{\mathrm{w}}}{F_{\text {decl. }}}\right| \sim \frac{2 \rho_{\mathrm{p} 0}\left(z_{\mathrm{rms}, \mathrm{A}}^{+}\right)^{2}}{\rho_{\alpha 0} U_{\mathrm{p} r} v_{\mathrm{A} 0}}\left(\frac{r_{\mathrm{A}}}{r_{0}}\right)^{2}\left(\frac{r}{r_{0}}\right)^{-1} .
$$

which is 0.25 at $r=r_{0}=0.29 \mathrm{AU}$ for $r_{\mathrm{A}}=10 R_{\odot}$, assuming that $z_{\text {rms,A }}^{+} \approx 300 \mathrm{~km} \mathrm{~s}^{-1}$ as in the numerical simulations of Perez \& Chandran (2013). The value of $\left|F_{\mathrm{w}} / F_{\text {decl. }}\right|$ decreases like $1 / r$ as $r$ increases beyond $0.29 \mathrm{AU}$.

These estimates show that the forces resulting from alphaparticle deceleration are significantly larger than $F_{\mathrm{G}}$ and $F_{\mathrm{w}}$. We also note that $F_{\mathrm{G}}$ and $F_{\mathrm{W}}$ act in opposite directions, so that their sum is smaller than either force individually. We conclude that the coasting approximation is reasonably accurate in the regions of the heliosphere on which we focus.

\section{CONCLUSION}

In this paper, we derive the rate $Q_{\text {flow }}$ at which energy is released by the deceleration of alpha particles in the solar wind. We also develop a solar-wind model that includes solar rotation, azimuthal flow, and the deceleration of alpha particles by two non-collisional mechanisms: plasma instabilities and the rotational force (Section 3.1). We use this model to evaluate $Q_{\text {flow }}$ in the fast solar wind at heliocentric distances between 0.29 and $4.2 \mathrm{AU}$.

The analytic expression we derive for $Q_{\text {flow }}$ is the first to account for the azimuthal velocities of the ions (cf Borovsky \& Gary 2014; Reisenfeld et al. 2001). We find that azimuthal flow makes an important correction to the energy-release rate and actually causes $Q_{\text {flow }}$ to become zero beyond a critical radius $r_{\text {crit }}$. In the fast solar wind, $r_{\text {crit }} \simeq 2.5 \mathrm{AU}$ in the heliographic equator. The value of $r_{\text {crit }}$ increases monotonically with heliographic latitude.

Our finding that $Q_{\text {flow }}=0$ at $r \geqslant r_{\text {crit }}$ relates to the way that plasma instabilities and the rotational force work together to decelerate alpha particles. At $r<r_{\text {crit }}$, the rotational force is unable to decelerate the alpha particles rapidly enough to keep the drift velocity $\Delta U_{\alpha \mathrm{p}}$ below the threshold value needed to excite the parallel-propagating $\mathrm{FM} / \mathrm{W}$ instability. As a consequence, differential flow excites FM/W waves, and resonant interactions between these waves and the alpha particles reduce $\Delta U_{\alpha p}$ as the plasma flows away from the Sun. These wave-particle interactions maintain $\Delta U_{\alpha \mathrm{p}}$ approximately at the marginally stable value, which decreases as $r$ increases. 
In contrast, at $r \geqslant r_{\text {crit }}$, the rotational force is sufficiently strong that it reduces $\Delta U_{\alpha \mathrm{p}}$ below the threshold value needed to excite instabilities. As a consequence, instabilities do not contribute to alpha-particle deceleration at $r \geqslant r_{\text {crit }}$. As mentioned above, $Q_{\text {flow }}=0$ at $r \geqslant r_{\text {crit }}$. Moreover, because of the corrections to $Q_{\text {flow }}$ resulting from the inclusion of azimuthal flow, $Q_{\text {flow }}$ decreases continuously to zero as $r$ increases from $0.29 \mathrm{AU}$ to $r_{\text {crit }}$. In Section 3.1.2, we also show that the previous treatments of the rotational force by McKenzie et al. (1979) and Hollweg $\&$ Isenberg (1981) are equivalent to the condition $Q_{\text {flow }}=0$, provided that $r$ is sufficiently large that other forces such as gravity can be neglected.

We present two types of numerical solutions to our model equations. First, we present a single solution that spans the radial range $0.29 \mathrm{AU}<r<1 \mathrm{AU}$ at zero heliographic latitude. Second, we present results from 1500 different solutions at heliographic latitudes ranging from $30^{\circ}$ to $80^{\circ}$, which span the radial range $1.5 \mathrm{AU}<r<4.2 \mathrm{AU}$. We compare these solutions to Helios and Ulysses observations, respectively.

Both types of solutions match the differential flow velocities $\Delta U_{\alpha \mathrm{p}}$ measured by Helios and Ulysses for choices of the alphaparticle temperature anisotropy $T_{\perp \alpha} / T_{\| \alpha}$ that are consistent with the observed values. However, the threshold value of $\Delta U_{\alpha \mathrm{p}}$ needed to excite the FM/W instability is sensitive to the value of $T_{\perp \alpha} / T_{\| \alpha}$. As a consequence, there are other profiles of $T_{\perp \alpha} / T_{\| \alpha}$ that are also consistent with the $T_{\perp \alpha} / T_{\| \alpha}$ observations for which our model does not accurately reproduce the measured values of $\Delta U_{\alpha p}$ (see Figure 7 and the Appendix). Thus, the comparison between our results and the observed $\Delta U_{\alpha \mathrm{p}}$ profile is not fully conclusive. Marsch \& Livi (1987) compared theoretical thresholds of the FM/W instability with observed alpha-particle beams in the solar wind. However, this study has not taken into account the effect of temperature anisotropies on the thresholds of beam-driven instabilities, which we find to be an important parameter.

As the alpha particles decelerate, bulk-flow kinetic energy is converted into wave energy and thermal energy. Because waves cascade and dissipate in the solar wind, we expect that $Q_{\text {flow }}$ is in effect a heating rate that results from alpha-particle deceleration. As we show in Figure 5, $Q_{\text {flow }}$ is comparable to the total empirical proton heating rate, denoted $Q_{\mathrm{p}}$, at $r \lesssim 0.42 \mathrm{AU}$, and $Q_{\text {flow }}$ exceeds the total alpha-particle heating rate at $0.29 \mathrm{AU}<r<1 \mathrm{AU}$, indicating that alpha-particle deceleration is an important heating mechanism in the inner heliosphere (cf Borovsky \& Gary 2014; Feldman 1979; Šafránková et al. 2013; Schwartz et al. 1981). Moreover, the increase in $Q_{\text {flow }} / Q_{\mathrm{p}}$ from $\simeq 1 / 4$ to $\simeq 1$ as $r$ decreases from $1 \mathrm{AU}$ to $0.29 \mathrm{AU}$ suggests that alpha-particle deceleration continues to be an important heating mechanism at $r<0.29 \mathrm{AU}$, the region that will be explored by Solar Probe Plus. In Figure 8, we show that $Q_{\text {flow }}$ is much less than $Q_{\mathrm{p}}$ at $r>1.5 \mathrm{AU}$, and that $Q_{\text {flow }}$ is comparable to the alpha-particle heating rate at 1.5 AU $<r<2.2 \mathrm{AU}$, which supports the argument of Reisenfeld et al. (2001) that alpha-particle deceleration is an important heating mechanism for alpha particles over at least the inner portion of the Ulysses orbit. On the other hand, we find that $Q_{\text {flow }}=0$ along the Ulysses trajectory at $r>3.3 \mathrm{AU}$, because at these radii the rotational force decelerates the alpha particles below the minimum drift speed needed to excite instabilities, and because deceleration by the rotational force does not reduce the bulk-flow kinetic energy of the plasma.
Table A1

Parameters in the Temperature Profiles in Equations (A1) and (A2)

\begin{tabular}{lcccc}
\hline \hline Parameter Set & $T_{\perp 0} / 10^{5} \mathrm{~K}$ & $T_{\| 0} / 10^{5} \mathrm{~K}$ & $\alpha_{\perp}$ & $\alpha_{\|}$ \\
\hline A & 7.0 & 8.0 & 1.40 & 1.20 \\
B & 6.0 & 9.0 & 1.37 & 1.155 \\
Section 4 & 7.0 & 8.0 & 1.37 & 1.155 \\
\hline
\end{tabular}

Regarding the azimuthal velocities of the ions, we find that the inclusion of differentially flowing alpha particles in our solar-wind model leads to a substantial increase in the azimuthal velocities of both alpha particles and protons, $U_{\alpha \phi}$ and $U_{\mathrm{p} \phi}$, relative to zero-torque solutions in which alphaparticles are neglected (Figure 3). The signs of $U_{\alpha \phi}$ and $U_{\mathrm{p} \phi}$ are the same at the effective co-rotation radius $r=r_{\text {eff }} \simeq 10 R_{\odot}$, but are opposite at the heliocentric distances exceeding 0.29 AU on which we focus.

Finally, our model of the spiral interplanetary magnetic field differs from Parker's (1958) in two ways. First, we assume that there is no net torque on the plasma beyond the effective corotation radius $r_{\text {eff }}$ (which we take to be located at $\left.r=r_{\text {eff }}=10 R_{\odot}\right)$. In contrast, Parker (1958) takes $U_{\mathrm{p} \phi}$ to be independent of $r$. Second, because the inclusion of differentially flowing alpha particles modifies $U_{\mathrm{p} \phi}$, it also modifies the angle $\psi_{B}$ between $\hat{\boldsymbol{r}}$ and $\boldsymbol{B}$. However, both of these effects are minor, and our value of $\psi_{B}$ is very close to Parker's (1958).

We thank Dan Reisenfeld, Kris Klein, Jean Perez, and Alfred Mallet for helpful discussions. This work was supported by grant NNX11AJ37G from NASA's Heliophysics Theory Program, NASA grant NNX12AB27G, NSF/DOE grant AGS-1003451, NSF grant AGS-1258998, and DOE grant DE-FG02-07-ER46372.

\section{APPENDIX \\ DEPENDENCE OF THE INSTABILITY THRESHOLDS $U_{\mathrm{t} 1}$ AND $U_{\mathrm{t} 2}$ ON $T_{\perp \alpha} / T_{\| \alpha}$}

The A/IC and FM/W instability thresholds $U_{\mathrm{t} 1}$ and $U_{\mathrm{t} 2}$ in Equations (24) and (25) depend on the temperature anisotropy of the alpha particles. To illustrate this dependence, we consider temperature profiles of the form

$$
T_{\perp \alpha}=T_{\perp 0}\left(\frac{r}{1 \mathrm{AU}}\right)^{-\alpha_{\perp}}
$$

and

$$
T_{\| \alpha}=T_{\| 0}\left(\frac{r}{1 \mathrm{AU}}\right)^{-\alpha_{\|}}
$$

with two new sets of parameters $T_{\perp 0}, T_{\| 0}, \alpha_{\perp}$ and $\alpha_{\|}$, denoted parameter sets A and B, whose values are given in Table A1 . Like the temperature profiles considered in Section 4, these new profiles are in approximate agreement with the Helios observations of Marsch et al. (1982b).

In Figure A1, we show the thresholds of both the A/IC and FM/W instabilities given in Equations (24) and (25) when we re-calculate the numerical solution presented in Section 4 using parameter sets A and B instead of Equations (50) and (51). In Figure A2, we show the profiles of $Q_{\text {flow }}$ in these new solutions. We find that the $U_{\mathrm{t} 1}, U_{\mathrm{t} 2}$, and $Q_{\text {flow }}$ profiles for parameter set A are similar to the corresponding profiles in 


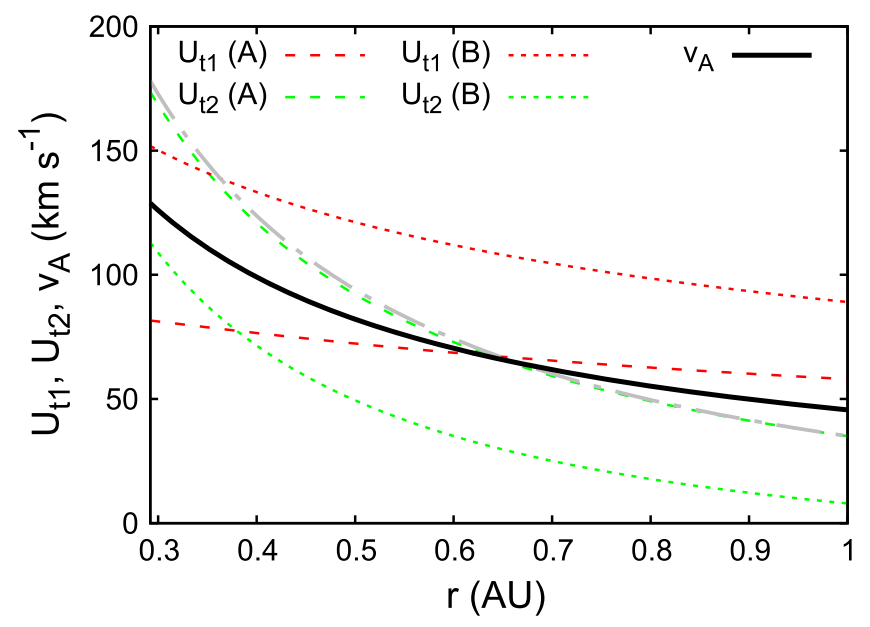

Figure A1. Radial profiles of $U_{\mathrm{t} 1}$ and $U_{\mathrm{t} 2}$ for parameter sets $\mathrm{A}$ and $\mathrm{B}$ in Table A1. The black line shows the profile of the Alfvén speed $v_{\mathrm{A}}$. The gray dash-dotted line shows our original solution from Section 4.

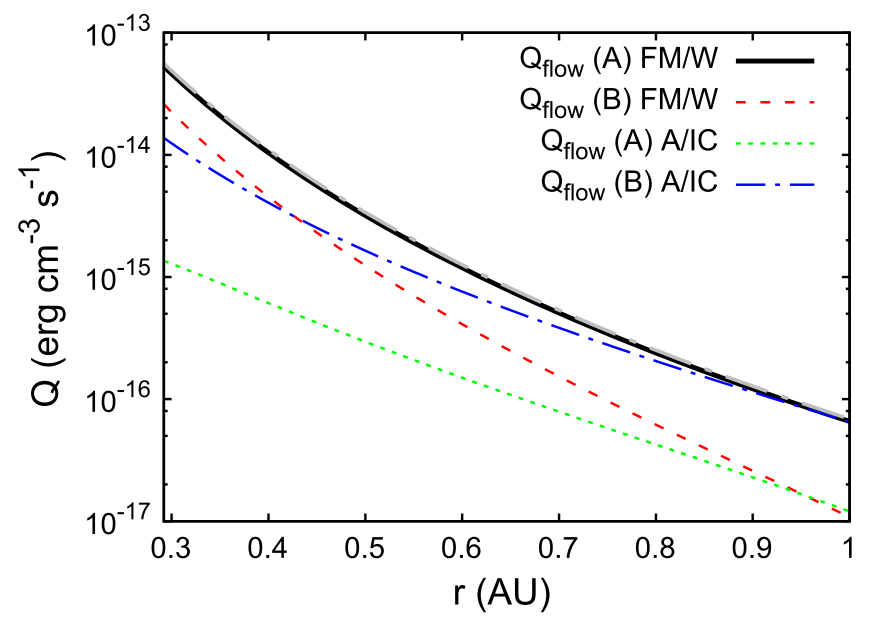

Figure A2. Radial profiles of $Q_{\text {flow }}$ for parameter sets A and B under the assumptions that $\Delta U_{\alpha \mathrm{p}}=U_{\mathrm{t} 1}(\mathrm{~A} / \mathrm{IC})$ and $\Delta U_{\alpha \mathrm{p}}=U_{\mathrm{t} 2}(\mathrm{FM} / \mathrm{W})$. The gray dash-dotted line shows our original solution from Section 4.

Section 4, but the profiles for parameter set B differ significantly. Thus, the $T_{\perp \alpha}$ and $T_{\| \alpha}$ profiles are an important source of uncertainty in our model.

For completeness, we also show in Figure A2 the values of $Q_{\text {flow }}$ under the (unrealistic for the reasons given in Section 3.1.1) assumption that

$$
\Delta U_{\alpha \mathrm{p}}=U_{\mathrm{t} 1} .
$$

Given Equation (A3), the value of $Q_{\text {flow }}$ for parameter set A is significantly smaller than in our original solution in Section 4. For parameter set B, Equation (A3) leads to a value of $Q_{\text {flow }}$ that is smaller than in the model presented in Section 4 at small $r$.

\section{REFERENCES}

Araneda, J. A., Viñas, A. F., \& Astudillo, H. F. 2002, JGR, 107, 1453 Bale, S. D., Kasper, J. C., Howes, G. G., et al. 2009, PhRvL, 103, 211101 Balogh, A., Marsden, R. G., \& Smith, E. J. 2001, The Heliosphere near Solar Minimum: The Ulysses Perspective (Berlin: Springer)
Bame, S. J., Asbridge, J. R., Feldman, W. C., \& Gosling, J. T. 1977, JGR, 82,1487

Barnes, A. 1981, in Solar Wind Conf. Ser. 4 ed. H. Rosenbauer (Garching: Max-Planck-Institute für Aeronomie), 326

Borovsky, J. E., \& Gary, S. P. 2014, JGR, 119, 5210

Bourouaine, S., \& Chandran, B. D. G. 2013, ApJ, 774, 96

Bourouaine, S., Verscharen, D., Chandran, B. D. G., Maruca, B. A., \& Kasper, J. C. 2013, ApJL, 777, L3

Chandran, B. D. G. 2010, ApJ, 720, 548

Chandran, B. D. G., Dennis, T. J., Quataert, E., \& Bale, S. D. 2011, ApJ, 743, 197

Chandran, B. D. G., \& Hollweg, J. V. 2009, ApJ, 707, 1659

Chandran, B. D. G., Li, B., Rogers, B. N., Quataert, E., \& Germaschewski, K. 2010, ApJ, 720, 503

Chandran, B. D. G., Verscharen, D., Quataert, E., et al. 2013, ApJ, 776, 45

Chaston, C. C., Bonnell, J. W., Carlson, C. W., et al. 2004, JGR, 109, 4205

Chen, L., Lin, Z., \& White, R. 2001, PhPl, 8, 4713

Chew, G. F., Goldberger, M. L., \& Low, F. E. 1956, RSPSA, 236, 112

Cranmer, S. R., Matthaeus, W. H., Breech, B. A., \& Kasper, J. C. 2009, ApJ, 702, 1604

Denton, R. E., Anderson, B. J., Gary, S. P., \& Fuselier, S. A. 1994, JGR, 99 , 11225

Dewar, R. L. 1970, PhFl, 13, 2710

Dusenbery, P. B., \& Hollweg, J. V. 1981, JGR, 86, 153

Feldman, W. C. 1979, RvGSP, 17, 1743

Gary, S. P. 1993, Theory of Space Plasma Microinstabilities (New York: Cambridge Univ. Press)

Gary, S. P., Yin, L., Winske, D., et al. 2003, JGR, 108, 1068

Gary, S. P., Yin, L., Winske, D., \& Reisenfeld, D. B. 2000a, JGR, 105, 20989

Gary, S. P., Yin, L., Winske, D., \& Reisenfeld, D. B. 2000b, GeoRL, 27, 1355

Gazis, P. R., \& Lazarus, A. J. 1982, GeoRL, 9, 431

Goldstein, B. E., Neugebauer, M., Zhang, L. D., \& Gary, S. P. 2000, GeoRL, 27,53

Goodrich, C. C. 1978, PhD thesis, Massachusetts Institute of Technology

Hellinger, P., Matteini, L., Štverák, Š., Trávníček, P. M., \& Marsch, E. 2011, JGR, 116, 9105

Hellinger, P., Trávníček, P., Kasper, J. C., \& Lazarus, A. J. 2006, GeoRL, 33, 9101

Hellinger, P., \& Trávníček, P. M. 2008, JGR, 113, 10109

Hellinger, P., \& Trávníček, P. M. 2013, JGR, 118, 5421

Hollweg, J. V. 1974, JGR, 79, 1357

Hollweg, J. V., \& Isenberg, P. A. 1981, JGR, 86, 11463

Hollweg, J. V., \& Isenberg, P. A. 1983, JGR, 88, 7253

Hollweg, J. V., \& Isenberg, P. A. 2002, JGR, 107, 1147

Hollweg, J. V., \& Lee, M. A. 1989, GeoRL, 16, 919

Hollweg, J. V., Verscharen, D., \& Chandran, B. D. G. 2014, ApJ, 788, 35

Isenberg, P. A., \& Hollweg, J. V. 1982, JGR, 87, 5023

Isenberg, P. A., \& Hollweg, J. V. 1983, JGR, 88, 3923

Isenberg, P. A., \& Vasquez, B. J. 2007, ApJ, 668, 546

Isenberg, P. A., \& Vasquez, B. J. 2009, ApJ, 696, 591

Johnson, J. R., \& Cheng, C. Z. 2001, GeoRL, 28, 4421

Kasper, J. C., Lazarus, A. J., \& Gary, S. P. 2002, GeoRL, 29, 1839

Kasper, J. C., Lazarus, A. J., \& Gary, S. P. 2008, PhRvL, 101, 261103

Kasper, J. C., Maruca, B. A., Stevens, M. L., \& Zaslavsky, A. 2013, PhRvL, 110, 091102

Lamarche, L. J., Vasquez, B. J., \& Smith, C. W. 2014, JGR, 119, 3267

Li, B., Habbal, S. R., \& Li, X. 2007, ApJ, 661, 593

Li, B., \& Li, X. 2006, A\&A, 456, 359

Li, B., Li, X., \& Labrosse, N. 2006, JGR, 111, 8106

Li, X., \& Habbal, S. R. 2000, JGR, 105, 7483

Lu, Q. M., Xia, L. D., \& Wang, S. 2006, JGR, 111, 9101

Mariani, F., Villante, U., Bruno, R., Bavassano, B., \& Ness, N. F. 1979, SoPh, 63,411

Marsch, E. 1986, A\&A, 164, 77

Marsch, E., Ao, X.-Z., \& Tu, C.-Y. 2004, JGR, 109, 4102

Marsch, E., Goertz, C. K., \& Richter, K. 1982a, JGR, 87, 5030

Marsch, E., \& Livi, S. 1987, JGR, 92, 7263

Marsch, E., Muehlhaeuser, K. H., Rosenbauer, H., \& Schwenn, R. 1983, JGR, 88, 2982

Marsch, E., \& Richter, A. K. 1984, JGR, 89, 5386

Marsch, E., Rosenbauer, H., Schwenn, R., Muehlhaeuser, K.-H., \& Neubauer, F. M. 1982b, JGR, 87, 35

Marsch, E., Schwenn, R., Rosenbauer, H., et al. 1982c, JGR, 87, 52

Maruca, B. A., Kasper, J. C., \& Bale, S. D. 2011, PhRvL, 107, 201101

Maruca, B. A., Kasper, J. C., \& Gary, S. P. 2012, ApJ, 748, 137

Matteini, L., Hellinger, P., Goldstein, B. E., et al. 2013, JGR, 118, 2771 
Matteini, L., Landi, S., Hellinger, P., et al. 2007, GeoRL, 34, 20105

McChesney, J. M., Stern, R. A., \& Bellan, P. M. 1987, PhRvL, 59, 1436

McComas, D. J., Barraclough, B. L., Funsten, H. O., et al. 2000, JGR, 105, 10419

McKenzie, J. F., \& Axford, W. I. 1983, JGR, 88, 7251

McKenzie, J. F., Ip, W.-H., \& Axford, W. I. 1979, Ap\&SS, 64, 183

McKenzie, J. F., \& Marsch, E. 1982, Ap\&SS, 81, 295

Mestel, L. 1968, MNRAS, 138, 359

Miyake, W., \& Mukai, T. 1987, P\&SS, 35, 185

Ofman, L., Gary, S. P., \& Viñas, A. 2002, JGR, 107, 1461

Parker, E. N. 1958, ApJ, 128, 664

Perez, J. C., \& Chandran, B. D. G. 2013, ApJ, 776, 124

Pizzo, V., Schwenn, R., Marsch, E., et al. 1983, ApJ, 271, 335

Press, W. H., Teukolsky, S. A., Vetterling, W. T., \& Flannery, B. P. 1992, Numerical Recipes in FORTRAN. The Art of Scientific Computing (New York: Cambridge Univ. Press)

Reisenfeld, D. B., Gary, S. P., Gosling, J. T., et al. 2001, JGR, 106, 5693
Revathy, P. 1978, JGR, 83, 5750

Samsonov, A. A., Alexandrova, O., Lacombe, C., Maksimovic, M., \& Gary, S. P. 2007, AnGp, 25, 1157

Samsonov, A. A., \& Pudovkin, M. I. 2000, JGR, 105, 12859

Scarf, F. L., \& Fredricks, R. W. 1968, JGR, 73, 1747

Schwartz, S. J., Feldman, W. C., \& Gary, S. P. 1981, JGR, 86, 541

Schwartz, S. J., \& Marsch, E. 1983, JGR, 88, 9919

Sharma, P., Hammett, G. W., Quataert, E., \& Stone, J. M. 2006, ApJ, 637,952

Thieme, K. M., Marsch, E., \& Rosenbauer, H. 1989, JGR, 94, 2673

Tu, C.-Y., \& Marsch, E. 1995, SSRv, 73, 1

Šafránková, J., Němeček, Z., Cagaš, P., et al. 2013, ApJ, 778, 25

Verscharen, D., Bourouaine, S., \& Chandran, B. D. G. 2013a, ApJ, 773, 163

Verscharen, D., Bourouaine, S., Chandran, B. D. G., \& Maruca, B. A. 2013b, ApJ, 773, 8

Verscharen, D., \& Chandran, B. D. G. 2013, ApJ, 764, 88

Weber, E. J., \& Davis, L., Jr. 1967, ApJ, 148, 217 